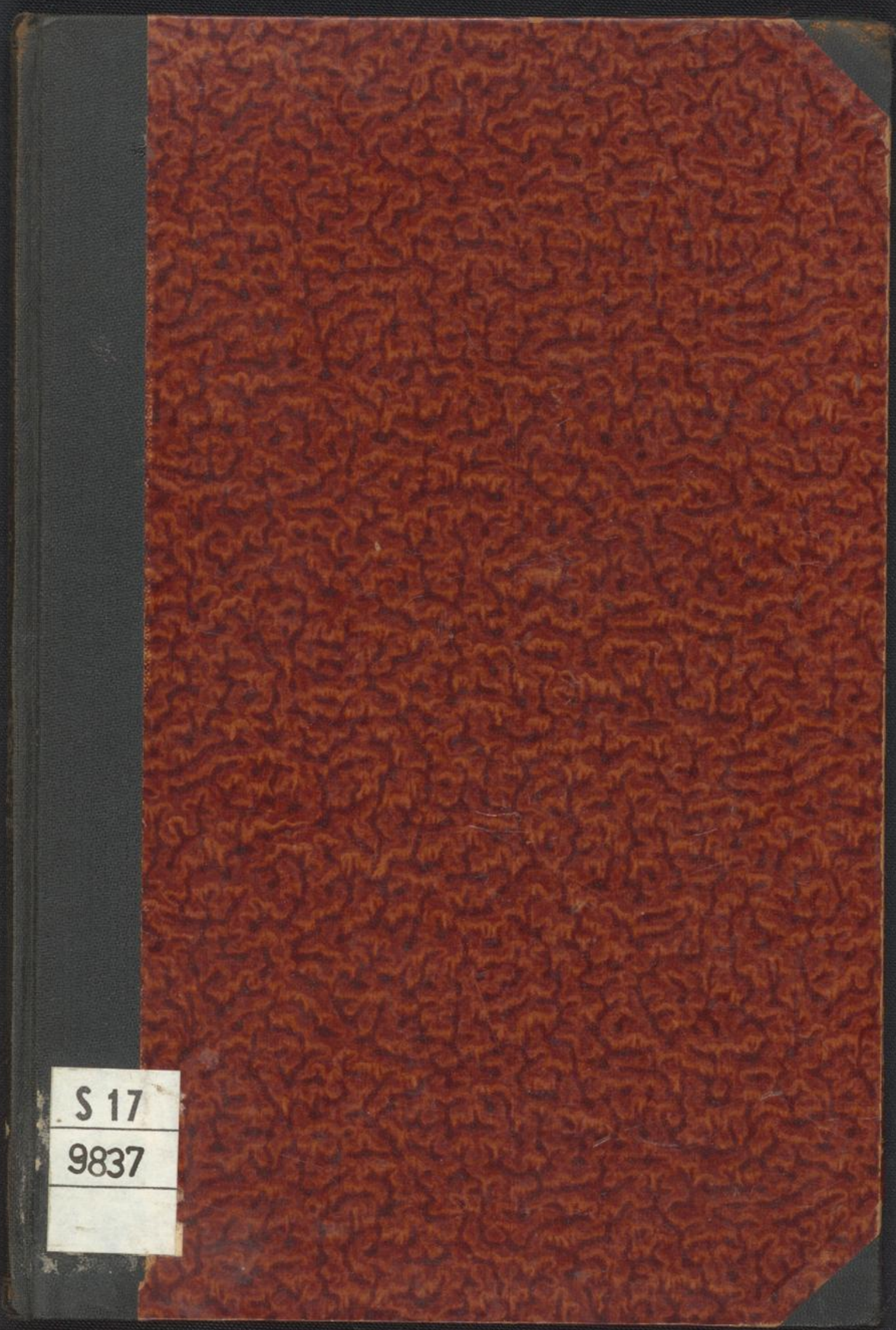




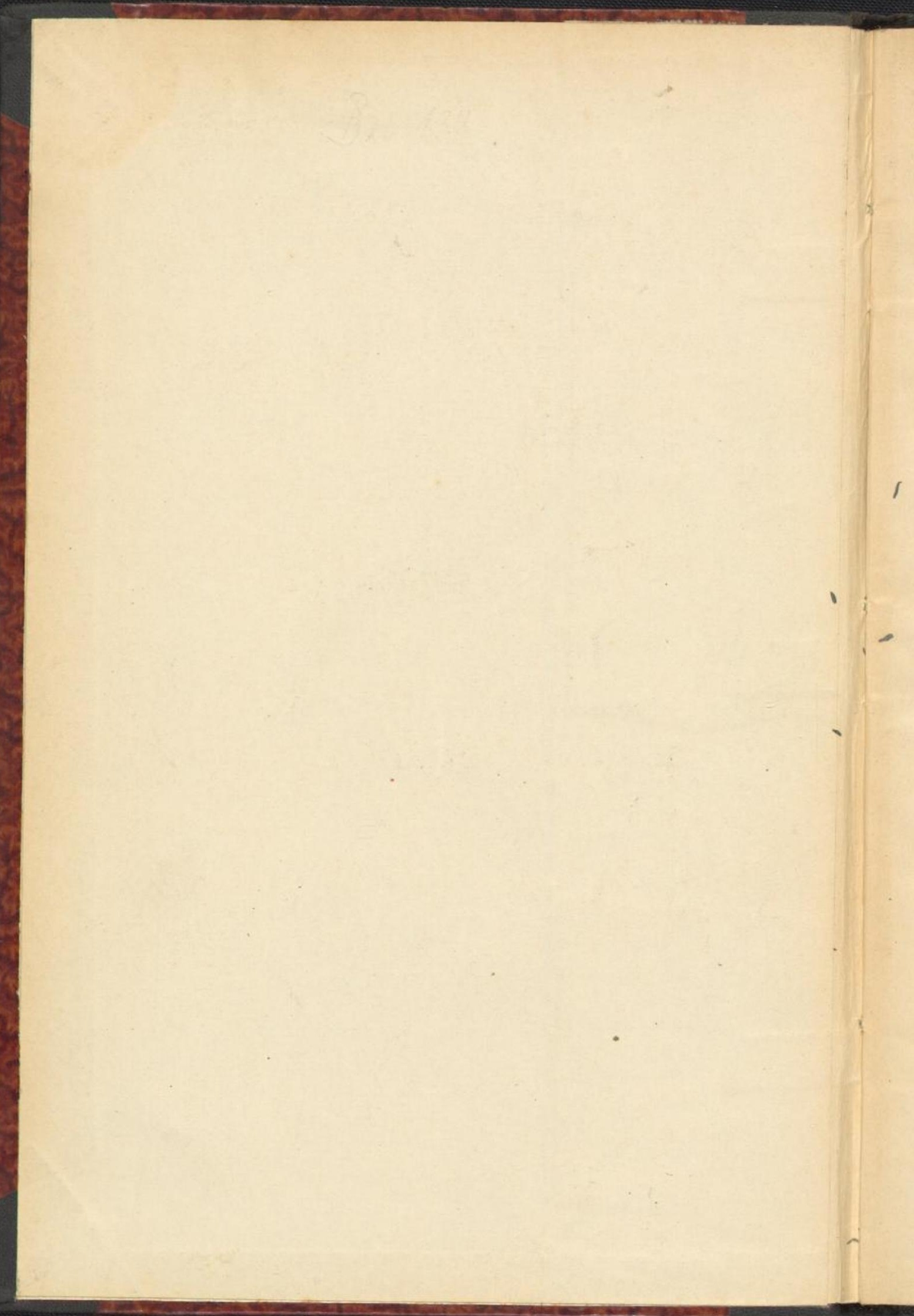




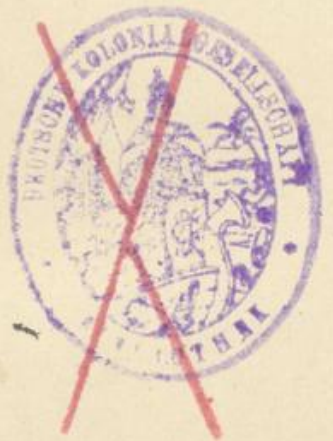

IIy UB Iniversitatsbibliothek Johann Christian Senckenbera $D=G$ 


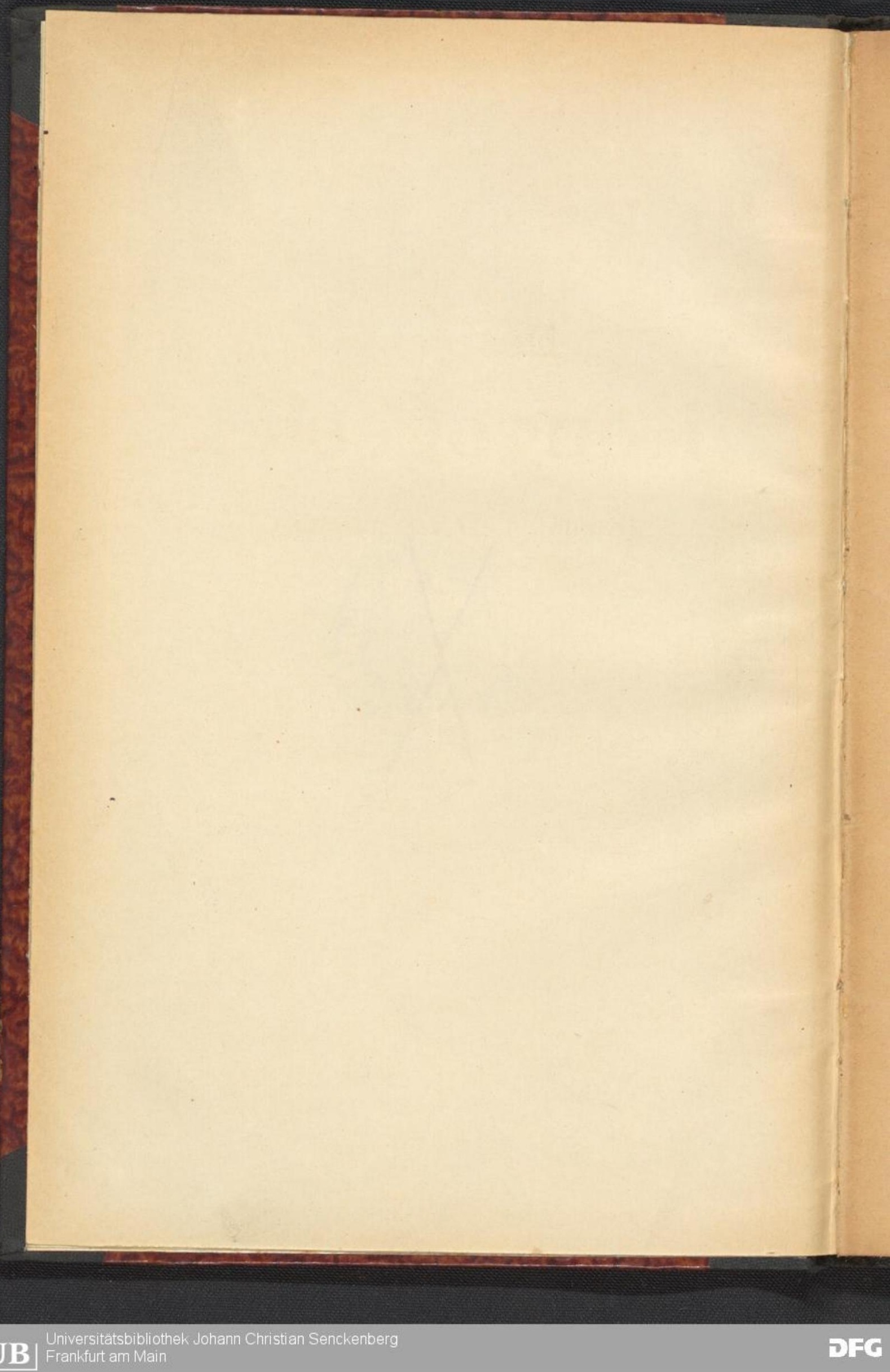




\section{Das neue \\ Kongogebiet (Kamerun, Deutschkongo)}

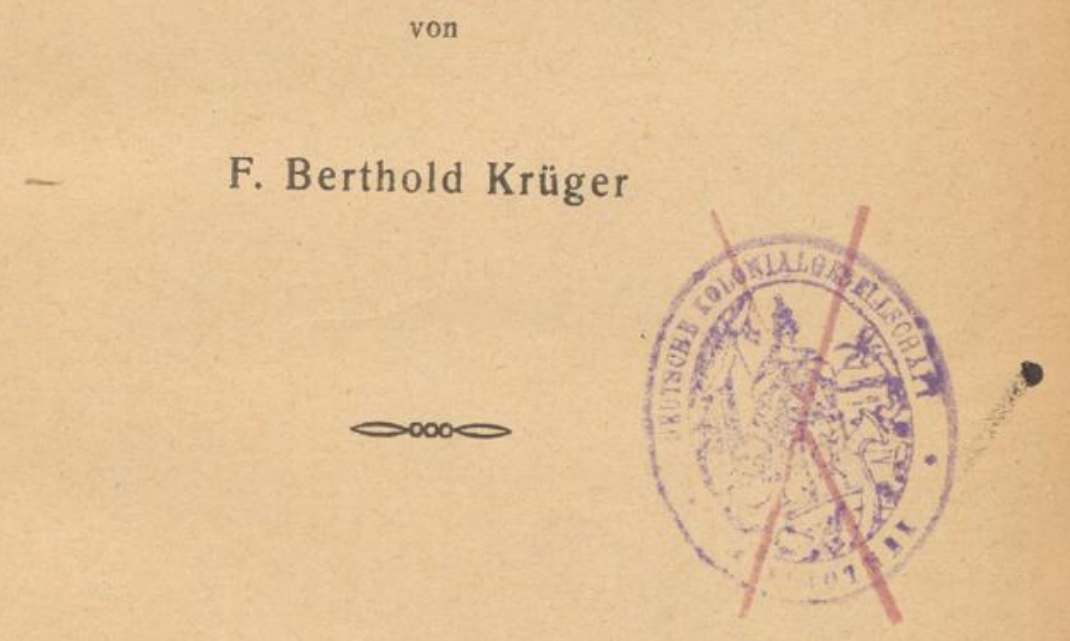

Leipzig

Ernst Marré's Verlag

(Orient-Verlag)

$1 / 12$ 


\section{S17/9837}

Stadt-u. Univ.-Bibl.

Frankfurt/Main

Druck von Arno Theuerkorn, Leipzig

\section{$401570 \times 1$}




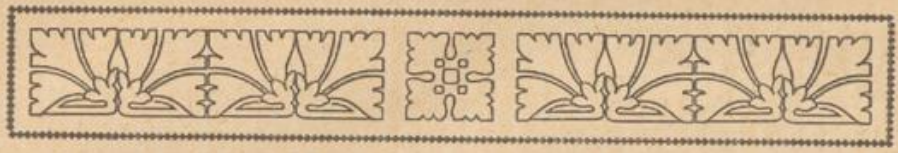

I.

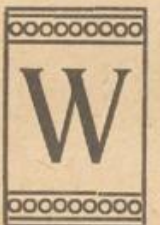

ir wollen in den nachstehenden Zeilen nicht Kritik üben, was besser sei: Marokko oder Kongo, sondern wir wollen von dem jetzigen Stand ausgehen: Deutsch-Kongo ist gegeben. Was können wir nun durch eine systematische Arbeit für die Allgemeinheit und für die Nation damit erzielen und was bietet oder kann das neue Kolonialland dem einzelnen Kolonisten und Kaufmann bieten? Viel springt freilich dabei nicht heraus, wenigstens nicht in absehbarer Zeit; das aber schließt nicht aus, daß wir später eine gewinnbringende Kolonie daraus schaffen können. Daß die Erwerbung des Entenschnabels von Nordost-Kamerun zum Territoire militaire des pays et protectorats du Tsad den Franzosen gelegen kommen mußte, bedarf wohl kaum der Erwähnung.

Die Bezeichnung Kongo entstammt nach einer Etymologie dem Namen seines Entdeckers, des Portugiesen Diego Cam (Cango), der 1484 die Mündung dieses großen Stromes erschloß und ihn für den König Johann II. von Portugal in Besitz nahm. An der Stelle, wo Diego Cam 1484 das Kongoland betreten hat, dem heutigen Kap Punta Padram, stand ein Steinpfeiler, der in portugiesischer und italienischer Sprache Kunde von der Entdeckung gab. Heute besteht von dem Denkmal nichts mehr.

Den Namen Kongo versuchte man andererseits je- 
doch etymologisch von einem Negerwort herzunehmen. Jedenfalls dürfte es weniger von dem portugiesischen Entdecker herzuleiten sein. Eine Lesart sagt, daß Kongo stammt von ko fngo gleich Heimat des Leoparden, eine andere stellt Kongo zu Rongo, Songo der Eingeborenensprachen, gleich Speer; meiner Ansicht nach liegt jedoch wohl eher das Wort kong Berg zu Grunde. Das alles sind teilweise nur Vermutungen und Ansichten, die für uns hier ohne Bedeutung sind. Sonst nennt man den Kongo Zaire, eine portugiesische Umbildung der Kintubezeichnungen Nsali, Zari u. a.

Wir hatten bereits früher in der Gegend ein deutsches Schutzgebiet, nämlich Tschintschoscho (port. Chinchoxo), etwa $2 \frac{1}{2}$ Stunde von Landana entfernt, nördlich vom Kongo, wo Paul Güßfeld die deutsche Flagge hißte. Um 1873 wurde schon an der Expedition gearbeitet, die ein auffälliges Mißgeschick hatte. Das erste Schiff "Nigritia“ scheiterte an der Sierra Leoneküste, das zweite "Liberia" verscholl ebenfalls. Die Station in Ts chintschoscho erforderte außerdem hohe Unterhaltungskosten; als Verdienst rechnet man ihr $\mathrm{zu}$, daß die Expedition von hier erstmalig einen "Menschenaffen" lebend nach Europa brachte.

Ursprünglich lockte das Gerücht von Gold auch die Conquestadores nach der Kongo- und Gabunküste, dann war das Gebiet ein Feld des Reichtums durch Sklavenhandel geworden und später von den Franzosen $\mathrm{zu}^{*}$ NNiederlassungen und Ansiedlungen befreiter Sklaven benutzt. Daraus ist auch der heute blühende Gabunort Libreville hervorgegangen. Seit 1862 arbeitet dort die Hamburger Firma Wörmann mit Erfolg. Erst seit 1885 hat man der westafrikanischen-Küste größeres Interesse gewidmet, seit dem Gründungsjahre des Kongostaates.

Die ersten gründlichen Bearbeiter des Kongo und des Kongogebietes waren Livingstone und nach ihm Stanley. Stanley, der Bula-Matari „Felsenbrecher" der 
Neger (ob diese Bezeichnung zutrifft oder übertrieben ist, soll festzustellen unsere Aufgabe nicht sein) schrieb in der Vorrede zu seinem "Kongo": Die jungen Leute werden Beobachtungen finden, kuhne Kaufleute werden erfahren, wie man eigentlich afrikanische Märkte zu betrachten hat, und jene, die gezögert haben, ein Absatzgebiet in den Tropen zu suchen, werden das bisher falsch beurteilte Klima verstehen lernen müssen.

Jeder muß sich also selbst der Lehrmeister sein, denn alle Ratschläge werden kaum genügen, das zu ersetzen, was eigene Erfahrung lehrt, namentlich hier im Kongogebiet. Wie groß dieser Strom ist, zeigt seine Messung. Der Kongo ist am südlichen Zipfel unseres Gebietes verhältnismäßig sehr tief und etwa $7 \mathrm{~km}$ breit. Wir haben vorläufig nur zwei Zipfel, die an dem großen Strom heranreichen. Mit diesen beiden Zipfeln läßt sich naturgemäß nicht viel anfangen, obwohl sie breit sind und bis zum schiffbaren Kongo und Ubangi sich erstrecken. Nun werden Bahnverbindungen zu schaffen sein, die an die Flüsse gehen. Auf Verbindungen hat Frankreich entschieden bei der Kompensation geachtet; daß wir mit Bahnbauten möglichst französisches Gebiet vermeiden werden und müssen, liegt auf der Hand. In der Absteckung der neuen Grenzen, so schreibt der Temps, hat die französische Regierung besonders Wert darauf gelegt, daß die Verbindungslinien zwischen den verschiedenen Teilen unseres afrikanischen Reiches nicht gestört werden. Wenn man absieht von drei Kilometern am Kongo und vier Kilometern"am Ubangi, beherrscht der französische Besitz ununterbrochen diese beiden Flußstraßen, und Frankreich wird imstande sein, über seinen Besitz nicht nur theoretisch, sondern auch praktisch zu verfügen. Außerdem erhält Frankreich neue Möglichkeiten, um die Linien am Benue auszunutzen und behält für seine Telegraphen, seine Eisenbahnen und 
selbst für seine Truppen das Recht, das deutsche Territorium zu durchschreiten.

Im Kongo wie Ubangi liegen neben Sandbänken bezw. unbedeutenden Schnellen auch noch kleinere Inseln vorgelagert. Ueber das Hoheitsrecht auf ihnen ist man sich noch nicht klar, obwohl die Hoheit über die Inseln sich nach der Regel des Uferbesitzes richtet. Die dem deutschen Ufer vorgelagerten Inseln gehören zum deutschen Gebiet, die dem belgischen (Kongostaat-)Ufer vorgelagerten Inseln zum belgischen Gebiet. Große Bedeutung kommt vielleicht diesen Flußinseln nicht zu, obwohl Ansiedlungen darauf in erster Linie leicht und rentabel sind. Doch hat man hier auch die Geologie zu Rate zu ziehen, nach ihrer Antwort erst wird die Lösung von seiten des Kolonisten ins Auge zu fassen sein. Hinsichtlich des Landerwerbs am Kongo und Ubangi und der Frage des Flußbesitzes kann man den unbestritten völkerrechtlichen Grundsatz sich beziehen, daß dem Besitzer des Ufers auch die Hoheit über den Fluß zufällt, wobei nur bestritten ist, ob die Grenze durch die Flußmitte oder den Talweg gebildet wird. Bei den der internationalen Schifffahrt eröffneten Flüssen, mit denen wir es zu tun haben, ist diese Streitfrage aber ohne Bedeutung. Die Schiffbarkeit an einzelnen Stellen südlich dieses Punktes durch Trockenheit unterbrochen. Für solche Fälle trifft die Bestimmung Vorsorge, daß das Gebiet des anderen Teiles zur Umgehung von Trockenstellen auf dem Landwege benutzt werden darf. $\mathrm{Ob}$ wir in der Lage sein werden, unsere Eisenbahn gerade in dem Zipfel an den Ubangi zu bringen, läßt sich heute noch nicht sagen. Wir haben auf alle Fälle das Recht, unsere Bahnen durch französisches Gebiet weiterzubauen. Dazu bedarf es der Verständigung mit Frankreich. Wir können also auch die Bahn an irgendeinem Punkt, der uns paßt, an den Ubangi bringen. Der 
Ort, wo die Schiffbarkeit des Ubangi aufhört, liegt jedoch noch viele Kilometer nördlich am Strome, sodaß wir von einer Möglichkeit, daß auch am deutschen Ubangiufer einmal die Schiffbarkeit durch physikalische Einwirkungen aufhören könnte, ganz absehen dürfen.

Die Regierung (Kolonialamt) hat über die Bewertung der Neuerwerbungen im Verhältnis zum abgetretenen Gebiet eine D enks chrift herausgegeben; wir greifen des Oeftern darauf zurück, sofern es uns im Interesse dieses Heftes nötig erscheint. Das hat zunächst für die Abgrenzung und die Grenzregulierung statt. Bei der Grenzführung ist zu bedauern, daß das Prinzip der natürlichen Grenzen nicht überall durchgeführt ist. Demgegenüber ist aber zu bemerken, daß besonders vorgesehen ist, daß die Vermessungskommission so weit wie möglich die Grenzen den natürlichen Verhältnissen des Geländes (Flußläufen, Wasserscheiden) und den Stammeszusammengehörigkeiten anpassen soll. Es liegt in der Absicht beider Parteien, daß die Kommissare von dieser Befugnis den weitgehendsten Gebrauch machen.

Andererseits kann nicht verkannt werden, daß die Grenze, so wie sie jetzt vereinbart ist, für uns große Vorteile bietet: Die Grenze läuft den Kandeka, Bokiba, Likuala abwärts, Sanga und Likuala aufwärts, dann ungefähr auf der Wasserscheide zwischen Sanga und Ubangi zum Lobaye, den Lobaye abwärts, dann unter Benutzung der Mbi ungefähr über die Wasserscheide zwischen Ubangi-Sanga- und Logonebecken zum Logone und diesen hinunter bis zu seiner Mündung. Diese Grenzführung bedeutet zweifellos eine Abrundung der Gestalt des Schutzgebietes Kamerun, da der sogenannte Entenschnabel weggenommen und der Entenhals bedeutend erweitert wird. Diese Verbreiterung der Würgestelle bedeutet eine ganz erhebliche Erleichterung für die Verwaltung der beiden Residenturbezirke, 
die durch die in unser Gebiet hineinspringende LereEcke bisher behindert war. Sie bringt ferner die durch die Tuburisümpfe hergestellte Verbindung zwischen dem Stromsystem des Benue-Mao Kebi und dem des Logone, so unvollkommen sie jetzt auch sein mag, ganz in deutschen Besitz. Gleichzeitig wird das Handelsgebiet für die Handelsniederlassungen in Garua bedeutend erweitert.

Als ein Schönheitsfehler in der Grenzführung müssen die im Osten und im Südosten vorspringenden Ecken angesehen werden. Betrachtet man sie aber nicht vom ästhetischen, sondern vom wirtschaftlichen Standpunkt, so kann ihr Vorteil nicht hoch genug eingeschätzt werden. Diese Ausläufer unseres Gebietes bringen uns in indirekte Verbindung mit der Hauptlebensader Zentralafrikas, dem Kongostrom, und seinen großen Nebenflüssen mit ihren mehreren tausend Kilometer Wasserstraßen. Sie eröffnen dadurch einerseits dem Schutzgebiet Kamerun zwei Tore für den Handel seines östlichen und südöstlichen Teils von und zu der Küste; andererseits gewähren sie Kamerun die Möglichkeit, bei weiterem Ausbau seines Wege- und Eisenbahnnetzes, in großen Teilen fremdländischen Kolonialbesitzes den Handel an sich zu ziehen und auf seine Bahnen zu lenken.

Angesichts dieser weitgehenden Entwickelungsmőglichkeiten wird man sich auch mit der Tatsache abfinden können, daß die weitere rationelle ErschlieBung der erworbenen Gebiete nicht ohne erhebliche Kosten möglich sein wird. Die Kosten müssen wir auf uns nehmen, wie wir sie willig und hoffnungsfreudig für unsere bisherigen Schutzgebiete auf uns genommen haben. Einen nicht unerheblichen Beitrag zur Bestreitung der Kosten wird uns die Konzessionsgesellschaft Sanga-Ubangi leisten, die allein an Pachtgebühr jährlich 170000 Francs und ferner 15 Prozent ihres be- 
deutenden jährlichen Reingewinns an den Staat abzugeben hat.

Das ganze Gebiet Congo Français, einschlieblich der Gabunbesitzungen überschreitet $700000 \mathrm{qkm}$ mit rund 5 Millionen Einwohnern; eine bedeutende Kolonisationsmöglichkeit erscheint sich hieraus zu ergeben.

Den Gabun und Ogowe, 1470 von den Portugiesen entdeckt, hat vorzïglich Oscar Lenz 1874 erforscht. Wenn er vielleicht auch die Afrikanische Gesellschaft, die ihn aussandte, nicht befriedigte, so sind seine Aufzeichnungen, die auch teilweise unser neues Kongogebiet streifen, von großem Werte. Seit 1885 wurde durch die internationale Konferenz das französische Kongogebiet von allen Mächten anerkannt und heute befindet sich wohl der größte Teil seines Handels trotz der Schikanen der Franzosen noch in englischen und deutschen Händen. Daraus ergibt sich auch eine eigenartige Stellung der Franzosen zu dieser Kolonie: Frankreich hat sein Kongogebiet nie hoch bewertet. Noch 1870 wollte man es sogar mit der britischen Kolonie Gambia austauschen.

Auf einen Faktor hat man jedoch ziemlich rücksichtslos geachtet. Im französischen Kongogebiet darf nur die französische Sprache gebraucht werden; diesem Befehl mußten sich auch die dort mit günstigemen $m_{e x}^{\text {E }} \mathrm{Er}$ folge arbeitenden englisch-amerikanischen Missionare, die ja auch in unserem südlichen Kamerun tätig sind, unterwerfen. Aber sonst verschiebt sich das Bild nicht zu Gunsten des französischen Staates. Es ereignete sich hier beispielsweise häufiger, daß die Regierung Konzessionen erteilte, die bereits anderen gegeben oder durch Priorität gesichert waren, andere wieder fanden ihr Gebiet nicht, es war Sumpfland, oder sie fanden das nach der Karte erworbene Land nicht, da dieser oder jener Fluß gar nicht oder an 
einer ganz andern Stelle existierte. Aus allen solchen Zerwürfnissen ist man also zu einer Kongomüdigkeit gekommen und zieht sich dadurch am glänzendsten heraus, - daß man das Land den Deutschen gibt, die nun ihrerseits das Labyrinth $\mathrm{zu}$ entwinden trachten müssen.

Nehmen wir alles in allem, so ist es aber selbstverständlich den Franzosen nicht leicht geworden, derartig große Gebietsstriche herzugeben. Der "Daily Chronicle" äußert: Die französische Regierung mußte bezahlen. Aber sie erhielt dafür bedeutende Vorteile, so daß sie sich über die von ihr gebrachten Opfer nicht zu beklagen braucht. Frankreich hat freie Hand an Marokko erhalten und daher in diesem Gebiet nichts von einer Nadelstichpolitik Deutschlands zu befürchten, wie sie Frankreich seinerzeit England gegenüber in der Nilgegend an den Tag gelegt hat. Die Aufgabe Frankreichs hat sich viel vereinfacht. Dennoch aber haben die Franzosen Glück, nicht zuletzt, da ihnen in Südafrika genug zu tun bleibt und sie das Mittelkongogebiet in absehbarer Zeit gar nicht kultivieren können: Durch die ihnen zugesicherte freie Hand in Marokko hat Frankreich immerhin überaus günstig abgeschlossen.

Wir aber dürfen das Neuland zur wirtschaftli chen Ausnützung nicht überschätzen, sondern wollen immer die bekannte graue Brille vorhalten, wer die Gefahr sucht, kommt keineswegs nun immer darin um. Tatkräftige Arbeit muß geleistet werden und zwar muß sie ihren Ausgang teilweise auch vom Kongo nehmen. In diesen Fällen würde freilich eine der gefürchteten Tropenkrankheiten in Kürze dem Kolonisten ein weiteres gedeihliches Arbeiten unmöglich machen oder der Urwald ihm jede wirtschaftliche Tätigkeit ohne große Geldquellen erschweren, wenn nicht geradezu unmöglich machen.

Die Franzosen haben heute noch, um ihre Ver- 
waltungsausgaben zu egalisieren, Zölle von 50 und mehr Prozent des Wertes der eingeführten Waren festgesetzt, mit einem derartig hohen Zoll wird man ein Land jedoch nie zu kolonisieren im Stande sein. Jedoch bewilligten die französischen Kammern 1883 über eine Millionen Franks und erst dadurch kam Leben in die Besitzung. Wenn jetzt Deutschland auch etwas für seine neue Kolonialerwerbung tut - und im andern Falle wäre die ganze Erwerbung überhaupt zwecklos - wird auch Frankreich sein Augenmerk auf seinen Besitz lenken und eine Rivalität in der Kolonisation entstehen, die dem Ganzen wie aber auch jedem einzeln nur von Nutzen sein wird.

Von großer Bedeutung, wie wir noch sehen werden sind die Kommunikationsmittel. Durch unser neues Gebiet geht eine der wichtigsten Karawanenstraßen; unsere Regierung hat uns selbst über diesen Vorteil belehrt, wenn sie schreibt: Einwendungen vom militärischen und politischen Standpunkt sind ganz besonders gegen die den Franzosen bezüglich einer Etappenstraße über den Benue-Mao Kebi-Logone eingeräumten Befugnisse erhoben worden. Die Sicherheit des nördlichen Teiles des Schutzgebietes sei dadurch gefährdet, daß französische Truppentransporte öfters durch unser Gebiet marschierten, und daß für die Etappe feste Stützpunkte gegeben seien. Demgegenüberist zu bemerken, daß die den Franzosen pachtweise eingeräumten Grundstücke (im Umfang von höchstens je $1 / 2$ Quadratkilometer) nur der Verproviantierung und der Stapelung von Material dienen, daher nicht befestigt und nur mit dem für die Beaufsichtigung und Bewachung der gestapelten Waren nötigen Personal nebst Familie und Diener dauernd besetzt sein dürfen; ferner, daß die Position der Franzosen in Wadai für absehbare Zeit eine exponierte bleiben wird, so daB sie kaum denken können, sich in einen ernsten Konflikt mit der Kame- 
runer Schutztruppe einzulassen. Der ganze Sinn des Marokkoabkommens ist doch aber der, daß es eine neue Aera der Verständigung und der Kooperation mit Frankreich auch auf kolonialem Gebiet einleiten soll. Darauf zielen im Abkommen selbst schon die Bestimmungen betreffend Eisenbahnbau durch FranzösischAequatorialafrika unter Beteiligung der deutschen Strekken nach gemeinsamer Uebereinkunft, die Mitbenutzung des französischen Telegraphen durch Deutschland, die Nichtbefestigung der Punkte an schiffbaren Flüssen, eine gemeinsame Bestimmung über die Flußschiffahrt und der freie Transit. Von demselben Geiste ist auch ein anderer Artikel diktiert, der den ungehinderten Durchzug französischer Truppen durch bestimmte französische Gebiete vorsieht. Von diesem Gesichtspunkte betrachtet muß man zugeben, daß die natürliche Etappenstraße für die wichtige und exponierte Stellung der Franzosen in Wadai durch deutsches Gebiet geht, sei es, daB sie dem Benue folgt, sei es, daß sie später, wenn einmal die Kamerun-Nordbahn das ganze Schutzgebiet durchquert, diese Bahn benutzt. Die jetzt von den Franzosen benutzte Etappenstraße ist unnatürlich; sie hat die doppelte Länge und ist sehr kostspielig. Wenn wir den Franzosen die für sie so wertvolle kürzere Etappenstraße, die wir ihnen übrigens für einen eiligen Truppentransport mit Rücksicht auf die gefährliche Lage, in der sie sich im vorigen Jahre in Wadai befanfanden, schon einmal zugestanden haben, dauernd öffnen, so leisten wir ihnen damit einen großen Dienst, den sie dauernd anerkennen müssen und werden. Die guten Beziehungen zwischen den beiden Mächten werden hierdurch gefördert werden. Die Einräumung von kleinen Grundstücken an eine fremde Macht im Wege der Pacht zur Ueberwindung von nichtschiffbarenStrecken sonst schiffbarer Flüsse und zur Erleichterung des Schiffverkehrs hat ubrigens sein Vorbild in dem eng- 
lich-französischen Vertrag vom 14. Juni 1898, in welchem England Frankreich je ein Grundstück am unteren und mittleren Niger unter ähnlichen Bedingungen, wie jetzt vorgesehen, verpachtet. Hervorzuheben ist hier, daB die französische Etappenstraße über den Benue zum weitaus größten Teil auf englichem Gebiet liegt und daß offenbar die Engländer keine politischen Bedenken gegen die Beförderung französischer Truppen durch englisches Kolonialgebiet haben, ferner, daß schon seit mehreren Jahren regelmäßig die Ablösungstransporte unserer Schutztruppenkompagnien in den Residenturbezirken über den Niger-Benue durch englisches Gebiet gehen.

Für uns Deutsche ergeben sich aber aus der Einräumung der französischen Etappenstraße auch direkte Vorteile: Politische: das Gefühl der Solidarität der Weißen gegenüber den Eingeborenen, das bei schlechten Beziehungen zwischen den europäischen Nationen leicht leidet, wird gestärkt. Besonderen Wert hat diese Tatsache in mohammedanischen Gebieten, wo der religiőse Fanatismus leicht zu umfangreichen, durch mehrere Kolonien gehenden Erhebungen führen kann. Wirtschaftliche Vorteile: In Wadai stehen allein 12 französische Kompagnien, worunter 2 Kompagnien Kamel= reiter und ein Artilleriedetachement, mit einer Gesamtstärke von 2400 Schwarzen, dazu das weiße Personal an Offizieren und Unteroffizieren, die mit einer Verpflegung fast auf die Nachschübe von Europa angewiesen sind. Das ungefähr am Endpunkt der Schiffbarkeit des Benue gelegene Garua wird daher bei einigermaßen geschicktem Vorgehen unserer Kaufleute als Handelsplatz einen großen Aufschwung nehmen, die wirtschaftliche Erschließung des nördlichen Teiles von Kamerun auf seine natürlichen Reichtümer erhält dadurch einen kräftigen Ansto $B$ und dauernde erhebliche Forderung (vor allem kommt hier die Viehzucht in Frage), 
wodurch die Aussichten auf Rentabilität und damit auf Erbauung der Kameruner Nordbahn steigen.

Die Einräumung der Etappenstraße ist also anzusehen als ein Stück internationaler Verkehrspolitik, einer Politik, deren Aufgaben in der Vergangenheit so gut wie !noch gar nicht an uns herangetreten sind, weil ihre Verwirklichung im allgemeinen einen höheren Entwicklungsstand unseres kolonialen Verkehrs- und namentlich Eisenbahnwesens voraussetzt, als wir ihn bis heute erreicht haben; einer Politik aber, die in kommenden Zeiten unsere Aufmerksamkeit in starkem Maße auf sich gelenkt zu sehen verdient: die großen Verkehrslinien des afrikanischen Kontinents drängen seit geraumer Zeit über die Landesgrenzen der einzelnen Kolonien hinaus; aus dem britischen Süden, dem portugiesischen Osten und Westen stöBt die Schiene in die belgische Mitte vor, unsere eigene ostafrikanische Zentralbahn wird, wenn sie demnächst den Tanganikasee erreicht, ihre Fortsetzung am anderen Ufer in der belgischen Lukugabahn erhalten; die ägyptische Nordsüdmagistrale strebt ihrem Zusammenschluß mit dem von Kapstadt nach Norden laufenden Schienenstrange entgegen; der gesamte Nordwesten Afrikas ist auf dem Wege, durch das eiserne Band des Bahngleises in festen Zusammenhang gebracht zu werden: kurz, Afrika zeigt die gleiche Entwicklung wie die übrigen Kontinente, die ein Netz hinüber- und herüberlaufender Bahnlinien gebaut und erst damit den heutigen Hochstand ihrer Wirtschaft ermöglicht haben. Nun liegt für jede unserer drei großen afrikanischen Kolonien die Sache so, daß ihr verkehrsgeographisches Hinterland beträchtlich weiter reicht, als die politischen Grenzen weisen, und daß sie somit die Anwartschaft haben (deren Vorteile auf der Hand liegen), Ausgang und Basis wichtiger und verkehrsreicher Interkolonialbahnen zu werden. Das gilt insbesondere von Kamerun mit seinem, den her14 
vorragenden Hafen Duala enthaltenden und verkehrsgeographisch so außerordentlich günstig gelegenen Kamerunästuar, dem innersten Teil der Einschnürung des Erdteils, die den einen Küstenpunkt der schmalsten Stelle Afrikas zwischen Atlantik und Roten Meer bezeichnet.

Die Ansichten der deutschen Regierung kann jedoch die Kolonialgesellschaft nicht teilen; sie nahm vielmehr folgende Resulution dagegen an: Es kann kein Zweifel darüber bestehen, daB die uns angeblich zugedachten Gebietsentschädigungen an den Kameruner Grenzen für uns keinen wertvollen Zuwachs bedeuten. Das im ganzen sumpfige und wenig fruchtbare Land bietet nicht viel und ist zum großen Teil einer erwerbsgierigen Konzessionspolitik ausgeliefert und unter der dünnen Bevölkerung wütet die Schlafkrankheit. Wir würden uns ferner durch den Erwerb dieser Gebiete wesentliche finanzielle Lasten aufladen. Vor allen Dingen darf nicht die Rede sein von einer Abtretung Nordbornus der Kameruner Nordspitze, unseres einzigen Zuganges zum Tschadsee, wie sie von französischen Zeitungen als in Frage stehend hingestellt worden ist. Die starken und kriegstüchtigen Stämme dieser Striche nördlich des Benue haben nicht nur ihre eigene schon entwickelte und noch entwicklungsfähige Kultur, sondern sind vor allem aus vollem Herzen deutsch geworden und wollen deutsch bleiben. Im übrigen könnten wir schon aus dem Grunde nicht in eine Preisgabe der Kameruner Nordspitze willigen, weil die Völker in Mittelkamerun südlich des Benuestromes das mit Recht als Schwäche unsererseits auslegen würden.

Sollte das nicht etwas zu schwarz und trübe gesehen sein? Wir möchten es fast annehmen. Jedenfalls haben wir aber nun einmal das Danaergeschenk und müssen es zu verwerten suchen. Wir dürfen es aber nicht entwerten, wie eine Ansicht vorschlug, da- 
raus ein Deportationsland zu schaffen. Als Verbrecherkolonie kann man das neue Gebiet nicht verwenden, oder würde ein gleich ungünstiges Resultat wie die Portugiesen in Angola erzielen. Nicht überall kann man gute Resultate wie in Australien erwarten. Die Kolonien, wie Kamerun, Togo und auch Südwestafrika würden direkt entwertet werden.

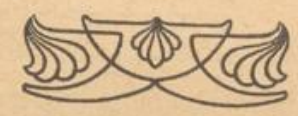




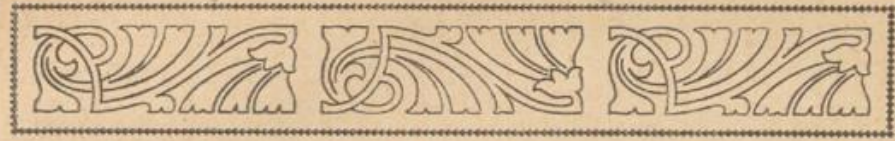

II.

000000000 ie Sterblichkeit ist in den Tropen erheblich, im Kongogebiet durch verschiedenartigste Einflüsse sogar sehr groß. Das ungesunde Klima erfordert viele Opfer. Wir unterschei000000000 den zwei Jahreszeiten, die trockene und die nasse, Winter und Sommer (nicht umgekehrt, wie man oft hört), in der letzten Periode steigt auch das Thermometer noch erheblich, namentlich tagsüber. Als spezielle Regenperiode kommt die Zeit vom Februar bis Mai und vom September bis Januar in betracht; die ausgesprochene Trockenzeit währt vom Juni bis zum August. In der Regenzeit, wenn der Regen nicht gerade tagelang anhält, ist es meist morgens schön, aber schon mittags beginnt es zu gießen und dies dauert dann bis in die Nacht hinein. Die mittlere Temperatur beträgt ca. $26^{\circ} \mathrm{C}$, die wärmste liegt im MärzApril, die kälteste im Juli-August.

Fast mit einem Tage kann man dann den Uebergang von Dürre zur Blütenpracht beobachten. Tagelang regnet es mitunter, daß es scheint, als würde alles fortgeschwemmt werden, was nicht niet- und nagelfest sitzt.

Das erste, was dem Neuling auffällt und womit er in Berührung kommt, sind die Uferränder; die des Meeres sind auch in Afrika überaus sandig, die der Flüsse hingegen bewachsen. Die Flußränder tragen

Krĩger Das neue Kongogebiet. 2 
Riedgräser, die uber $2 \mathrm{~m}$ hoch werden, sie treten als dichtes Gebüsch auf. Dann aber schließen sich ausgedehnte Savannen an, Grassteppen von großer Höhe und dickem Gestrüpp. Man nennt sie allgemein Kampinen. Diese Grasflächen können bei bewegter Luft mit den Wogen unserer Kornfelder verglichen werden, nur sind sie noch bedeutend höher und stachliger. Die einzige Abwechslung bilden darin die ziemlich nutzlosen Hyphaenepalme und der Segensbaum des schwarzen Erdteils, die Oelpalme, Elaeis guineensis. Man trifft die Oelpalme aber nicht nur vereinzelt, sondern auch in waldartigen Beständen.

Sind die Flußufer bewaldet, so findet sich meist darunter die Mangrove, die häufigste Art Rhyzophora Mangle mit ihren 2-4m großen Luftwurzeln aus weichem markigen Bestand. Ihre Stämme erreichen 15, ja selbst 30 m Höhe.

Wo deutsches Gebiet des südlichen Zipfels an den Kongo heranreicht, findet man einen selten schönen waldreichen Ufersaum, die Inseln davor haben ebenfalls herrlichen Baumwuchs. Auch umgestürzte Baumriesen finden sich in großer Zahl. Die Baumstämme schwimmen dann vielfach auf den Flüssen und versperren die Fahrt, hingegen begünstigen sie oft den Reisenden, da sie ihm das Uebersetzen über ein Wasser erleichtern.

Unter dem reichen Urwaldbestand finden sich viele wertvolle Bäume, darunter Drachenbäume, Palmenarten, Rotholz, Pterocarpus, das den Eingeborenen Schminke gibt und einen wichtigen Ausfuhrgegenstand bildet. Der Holzreichtum ist entschieden bedeutend, denn es kommen auf einen Quadratkilometer nicht selten 200 Stämme, vielfach 20 Meter hoch, oft kerzengrade, von Schlinggewächsen umgürtet; darunter finden sich ferner viele Teak- und Guajakbäume von hohem Wert. Auch zahlreiche andere Nutzhölzer gedeihen in 
großen Mengen, wie Diospyros Ebenholz, Boswellia Mahagoni.

Der Rizinusbaum kommt sehr gut fort und das gewonnene Rizinusöl findet zu guten Preisen Abnehmer. Ein häufiger Baum ist dann Artocarpus, der echte Brotfruchtbaum, der im Laufe der Zeit wohl zu veredeln wäre, eher als der Baobab, Adansonia. Seine Frucht, eine dickschalige grüne Apfelsine mit breiigem Mark, wird bereits als "Erfrischungshappen" genossen. Die Neger gewinnen durch Trocknen aus der Frucht ein Mehl, nehmen es aber durchgängig auch nicht gern. Leider nur spärlich sind Kokospalmen und Kautschuklianen vorhanden, letztere sind durch Raubzuige auf Gummi geradezu ausgeplündert, obwohl sie stellenweise im zentralen Afrika noch den größten Gewinn bringen und daher allgemein unter Kultur genommen werden. Dafür gedeihen viele andere Nutzpflanzen gut; wir erwähnen nur die Erdnuß, den Maniok und zahlreiche andere. Wir kommen auf die Kultur- und Nutzgewächse noch eingehend zurück, da sie einen Hauptteil der Verdienstmöglichkeiten für den Ansiedler und Kaufmann ausmachen.

Großes Raubwild ist verhältnismäßig selten, Büffel und Wildschweine kommen vor, ebenso verschiedene Antilopenarten. Sogar die nutzreiche Kuhantilope, Hartebeest wurde gemeldet. Affen sind zahlreich sowohl an Zahl wie an Art.

Von Vögeln interessieren vornehmlich die verschiedenen Papageien. Der Geier Gypohierax bildet auch in dem neuen Gebiete um die Ansiedlungen das Organ der Wohlfahrtspolizei.

Raubzeug erlegt man am besten mit Fallen der bekannten Raubtierfallenfabrik von R. Weber, Haynau, Schlesien, deren sog. Selbstschüsse und Fallen zum Lebendfang unübertroffen sind.

In den Flüssen finden sich Krokodile, die vielfach 
noch über $3 \mathrm{~m}$ lang werden, sie bilden für unbesonnene Badegäste eine arge Gefahr. Recht häufig ist die giftige grauschwarze Brillen- oder Speischlange, die ihren Giftspeichel einen Meter fortzuschleudern vermag. Auch die Naja haje findet sich. Diese Aspisoder ägyptische Brillenschlange ist eine Verwandte der gewöhnlichen Speischlange, Sepedo haemachates. Erste- re ist giftiger als letztere, da diese nie durch Giftausspeien dem Angegriffenen das Augenlicht rauben kann. Eigenartig bleibt hingegen, das die Speichel dieser weniger giftigen Schlangen (Colubriden) viel länger ihre Giftigkeit selbst bei hohen Temperaturen halten als die Sekrete der giftigeren Arten (Viperngift). Von anderen Giftschlangen werden namentlich noch die kurzen dicken Tigerschlangen gefährlich. Python Sebae, die afrikanische Riesenschlange ist 'recht häufig; sie wird 4-6 $\mathrm{m}$ lang und im Jugendstadium zum Rattenvertilgen auch freigehalten. Sie weiß Rattenlöcher besser als Katzen zu finden.

Die Moskitos, der Schrecken des Reisenden, bleiben im ganzen Afrika, wie in allen übrigen trop. Ländern eine große Plage, man kann sich ihrer nur mit vieler Mühe und Umsicht erwehren.

Wenn sie auch nachts nicht unter das feinmaschige Moskitonetz kommen können, verursachen sie doch ein arges Summen, daß man kaum Ruhe findet. Sie sind Uebertrager der gefährlichsten Tropenkrankheiten (Malaria). Die Stechmücken, Schnaken, Gelsen (Moskitos) gehören zu den verbreitesten Zweiflüglern. Beim Blutsaugen bringen die (weiblichen) Tiere, die in ihrem Körper auftretenden Keime pathogener Protozoen oder die Larven anderer Blut- oder Gewebsschmarotzer in die Gefäßbahnen der Menschen und erzeugen hier verschiedene Erkrankungserscheinungen, namentlich sind sie in feuchten, windstillen Tälern |mit Unterholz gefährlich. Sie treten vornehmlich in der Dämmerung 
auf und ruhen tagsüber unter den Blättern. Die Grundbedingung zu ihrer Existenz ist freilich nicht nur Feuchtigkeit, sondern auch stehendes, wenig bewegtes Wasser, sodaß wir sie selbst in den Blattachseln der Palmen, wenn diese auch nur noch wenig Wasser halten, allgemein finden.

Ganz besonders berüchtigt sind auch die Tsetsen, wie wir noch sehen werden.

Die Tsetsen, Glossinen, gehören zu den Muscinen, $z u$ denen auch unsere Stubenfliege gehört, sie sind nicht viel größer, ihre Mundteile sind zum Stechen eingerichtet. Sie machen eine vollkommene Entwickelung durch und zwar wird aus dem Ei eine fußlose Larve (Made), dann eine Tönnchenpuppe, also eine Puppe, bei der die erhärtete Madenhaut als Puppenhülle verwendet wird, und endlich eine fertige Fliege (Zungenfliege). Beim Stechen und Blutsaugen, flieBt „Speichel“ der Tsetsefliege in die Wunde und die Parasitübertragung ist vollzogen, da sich in ihm Trypanosomen finden.

Auch auf Heuschreckenplagen muß man vorbereitet sein. Oedipoda migratoria, die Wanderheuschrecke, sucht die dortigen Landstriche leider nur zu erfolgreich, wenn auch selten auf. In zwei bis drei Stunden fressen die Tiere alles kahl. Man schlägt die Heuschrecken zu Boden und sammelt sie. Die Neger braten sie in Palmöl, nachdem sie ihnen zuvor die häutigen Flügel und starren Beine abgerissen haben.

Zahlreiches kleine Ungeziefer verleidet den Kolonisten und Händler den Aufenthalt; auch die Negerwanzen stellen sich oft bei dem Weißen ein, es sind dies Argas, Ornithodoros Savignyi.

Ganz bekannt sind auch die Termiten-Ameisen. Termes bellicosus errichtet $30-40 \mathrm{ctm}$ hohe Bauten, geht aber nicht an Menschen, zerstört dafür umsomehr seine Waren. Man verscheucht die Termiten ziemlich 
sicher durch Petroleum. Die Termitenbauten zeigen sonderliche Formen, große Berge, Pilze, Türme oder Burgen.

Recht unangenehm macht sich auch der Sandfloh bemerkbar; Sarcopsylla (Rhynchoprion) penetrans bildet eine schreckliche Landplage; das Weibchen bohrt sich in die Haut des Menschen, (Zehen, Finger werden bevorzugt). Es legt Eier, dann beginnt der Schmerz, die Stelle schwillt an, herausschneiden hilft hier allein, wenn es frühzeitig vorgenommen wird, später stellen sich sonst schwere Verstümmelungen ein. Nur Reinlichkeit hilft prophylaktisch. Der Sandfloh selbst wird $1 / 2-1 \frac{1}{2} \mathrm{~mm}$ groB und ist rotbraun. Der Hinterleib schwillt beim Eierbilden bis zur GröBe eines Pfefferkorns an. Er fällt nicht nur Menschen sondern auch Hunde, Schweine, Hühner u. a. an. Selbst in der Freiheit wild lebende Tiere werden von ihm geplagt. Sarcopsylla wurde erst 1872 von Rio de Janeiro nach Afrika verschleppt; er ist hier jetzt so allgemein, wie ich ihn noch auf meinen Amerikareisen getroffen habe. Die Neger zeigen ein meisterliches Geschick, operativ diesen Schmarotzer herauszuholen und benutzen hierzu nur eine Nadel oder einen Holzstab. Gottseidank ist dieser Plagegeist nur auf sandige, möglichst trockene Länderstriche beschränkt. Jedenfalls ist aber als sicher anzunehmen, daB der Sandfloh jetzt in Afrika viel häufiger angetroffen wird als in seiner Heimat Amerika.

Wenig vertrauenerweckend liegen die Eingeborenen-Verhältnisse. Die Franzosen haben durch ungeeignete Behandlung eine Scheu eingeflöBt, die zur Abneigung sich steigerte und woran unsere Kolonisten auch schwer zu tragen haben werden. Dem Mißtrauen der Neger wird erst jede "Nahrung" entzogen werden müssen; das aber wird eine Sisyphusarbeit werden. Die Eingeborenen gehören jedenfalls zu den unangenehmsten Zugaben der Verwaltung; es bedarf diplomatischer Kunst hier Frieden zu halten. 
Die gröBte Untugend der Neger bleibt zweifellos die Unverschämtheit. Auf die Eingeborenen ist kein VerlaB; die Neger können nur für geringe Arbeiten verwendet werden, aber immer noch besser sind sie als die Mulatten, die Bastarde, die sich auch noch durch große Unbarmherzigkeit und Grausamkeit gegen die Neger auszeichnen.

Die Eingeborenen unter sich bekriegen sich vielfach; meist aber handelt es sich um unblutige, geringe Kämpfe. Verschiedene größere Stämme finden wir im neuen Kongolande. Zunächst die Fan, die jedoch vornehmlich mehr westlich wohnen bis in unser südliches Kamerun, und die Pongwe (Pongo). Beide Stämme sind erst wenig erforscht. Mit Sicherheit kann man jedoch annehmen, daß diese Völker sich verringern jedenfalls aber nicht zunehmen.

Ein besonders intelligentes Volk sind dann die Bateke, die auch viele Karawanen führen und dort, wo sie ansässig sind, etwas Ackerbau treiben. Man hat bei ihnen allgemein Wanderungen festgestellt wie bei den Fiote, dem Hauptvolke der Kongomündung. Wo Wanderungen vorkommen, sind sie naturgemäß durch Einflüsse aus Unterhalt, Verdrängung $u$. a. hervorgegangen.

Ein anderes Volk, die Ubangi oder Bafuru, besitzt großen Unternehmungsgeist. Es sind schön gebaute, kräftige Leute; auch sie drängen weiter und zwar nach Süden. Die Ubangi treiben Schiffahrt und Handel. Wir haben dann noch Ausläufer der Bakalai (südlicher Ogowe), der Batoka (oberer Sanga) und manche andere, auch Pygmäen wie die gelbbraunen Aschango (Abongo) finden sich in unserm neuen Kongogebiet.

Auf Kleidung legen alle Neger, die noch nicht unter ${ }$ Kultur" gestanden haben, wenig Wert, sodaß schon Stanley einen ${ }_{n}$ Altkleidermarkt" als sehr gewinnbringend empfahl. Hingegen wird auf Schmuck und Putz ein 
hoher Wert gelegt, dazu gehört auch das widerliche Einschmieren. Ein Einfetten des Körpers ersetzt die Kleidung und, obwohl es einen furchtbaren penetranten Geruch abgibt, ist es zweifellos mindestens ebenso vorteilhaft, als in diesen Gegenden volle Bekleidung zu tragen. Auch andere schlechte Eigenschaften haben die Neger unseres neuen Kolonialgebietes, sie „berauschen" sich gern an Palmwein, einer unangenehm säuerlich schmeckenden, milchig-trüben Flüssigkeit oder sie rauchen Liamba, Hanf (Cannabis, Haschisch). Daß hingegen die Neger auch an Heimweh erkranken können, erscheint uns fast unglaublich und doch ist es so; aber entgegen steht noch ein Appetit auf Menschenfleisch. Dem Kannibalismus wird noch Jahre lang im deutschen Kongolande, wenn auch nur heimlich gefröhnt werden.

Im Verkehr mit dem Neger muß der Weiße sich frei geben, Mißtrauen darf nicht aufkommen. Argwohn muß im Umgange mit den Eingeborenen unbedingt vermieden werden. Wenn wir wissen, daB in jeder Kolonie, nicht zuletzt in Deutschsüdwest, Kolonisten im Hinterhalt von Eingeborenen überfallen werden, um wieviel mehr besteht die Gefahr in einem Lande, deren Bewohner teilweise nicht nur noch dem Kannibalismus, sondern sogar auch dem Endokannibalismus huldigen. Auf Greueltaten der Eingeborenen müssen wir also noch lange gefaßt sein. Die Grausamkeiten, die uns unmenschlich erscheinen, nimmt der Neger durchgängig nach seinem Gefühlsleben weit leichter, denn er versteht es auch Leiden zu ertragen. Ein meuchlerisches Hinschlachten von Händlern und Ansiedlern wird noch Jahrzehnte lang zu erwarten sein, damit muß jeder Kolonist rechnen. Bei der Heimtücke des Bantu nutzen hier auch Strafexpeditionen in unserem Sinne nichts, da müßte schon ein wenig Grausamkeit hineinspielen. Im andern Falle wird eine Strafexpedition nie von großen objektiven Folgen begleitet sein. Der Neger 
ist dazu auch vielzusehr Augenblicksmensch und überrechnet nie die Folgen seiner Gelüste. $\mathrm{DaB}$ planvolle Verhetzungen von seiten anderer Stämme, Araber, ja Europäer nichtdeutscher Nation ihr übriges beitragen werden, muß sich jeder einzelne Ansiedlungslustige von vornherein sagen. Darum wird er mit heimtückischen Ueberfällen auf sein Eigentum, selbst auf größere Ansiedlungen rechnen, ganz gleich ob er sich freundschaftlich - deutsche Ansicht - oder streng - englische bezw. belgische Ansicht - zu den Eingeborenen stellen wird.

Reden - sicher auch eine Untugend der Eingeborenen, namentlich der Häuptlinge, die man allgemein als Kings, Könige bezeichnet. Jedes Beraten, Palaver (von parabola Wort) dehnt sich durch Geschwätz und Reden stundenlang aus und - man ist zu keinem Ziele gekommen. Die Negersprachen sind im allgemeinen klangreich. Die Sprachen des DeutschKongogebietes rechnet man zu den Fansprachen. Man zählt zu der Gruppe der Kintu, der Sprache der Bantu (der zentralafrikanischen Negervölker) u. a. vielleicht auch Kwala (Kwole), Itoni (Jetone), Mabea, Ngumba, Batyenga, Aduma, Jaunde, Koko, Wute. Immerhin aber kann man bei den noch ungenügenden linguistischen Kenntnissen von einem gleichen Sprachstamme nicht reden. Wir haben auch Verbindungen in einzelnen Sprachen, die keineswegs Kintuformen sein können. Die Sprachen haben hingegen mehr oder weniger alle eine auffällige Nasalierung, wir sprechen daher auch Fang; das Wort bedeutet jedenfalls „Mann“, e-Fam Mehrzahl ba-Fam nach Torrend, Vergleichende Grammatik „Der Mensch", von anderer Seite wurde die Bezeichnung Ba-huin gebraucht, das - etwas recht weit gegriffen an das Hottentotten (Nama) Khoin anklingen könnte, jedoch darf man aus Anklängen keine Etymologie konstruieren. Freilich spricht auch noch ein ethnographischer Standpunkt für diese „Verwandt- 
schaft"; wir haben gerade im neu erworbenen Kongogebiet Reste hellfarbiger, pygmäischer Neger, die ich für Einsprengungen von Urvölkern halte und die dann mit anderen, nicht zuletzt ost- und südafrikanischen Völkern zusammengebracht werden müßten. Die Linguistik kommt meiner Hypothese sehr nahe, näher als die Ethnographie.

Ich halte die Fansprachen von heute keinesfalls für eine einzige Gruppe; die verschiedensten Einflüsse machen sich da geltend. Ich erinnere beispielsweise nur an den im ganzen Hinterlande gebrăuchlichen Ausdruck für Europäer nasala, das reinarabischen Ursprungs ist. Ueberhaupt finden wir viele arabische Lehnwörter in den Sprachen, die durch Vermittlung einer andern Sprache, namentlich durch die lingua franca, die nordafrikanische Karawanensprache - das Hausa eingedrungen sind. Wir möchten daher jedem Interessenten das "Lehrbuch der Hausasprache " (Ernst Marré, Verlag, Leipzig, 2,20 Mk. postfrei) empfehlen, da in ihm eingehend über die afrikasprachlichen Verhältnisse gehandelt ist. Mit Literaturangaben der Fan- und PongweSprachen dienen wir weiter unten; selbstredend gibt es noch verschiedene andere Hilfsmittel, die aber bei einer kurzen Uebersicht nicht in Frage kommen können. Auch das Pongwe reiht man heute mit ziemlicher Sicherheit in die Fangruppe der Bantusprachen (Kintu). Es ist eine agglutinierende Sprache, welche durch Vorsetzen und Anfügen von Silben an den Stamm Bedeutungsveränderungen hervorruft. Wir reihen sie in die Kintu, die Sprachen der Bantu. So bilden wir die feinsten Sprachnüanzierungen, die selbst eine flektierende Kultursprache nicht wiederzugeben vermag; etwa aus einem Verbum formt man

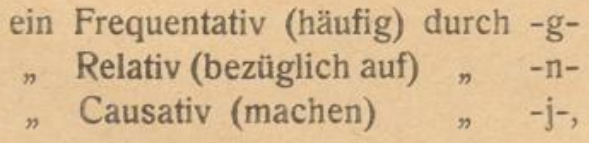


daher vom Infinitiv fanya fliehen z. B.: fanyinija jemand zur Flucht verhelfen, wörtlich: ihn fliehen machen (Relativ und Causativ). Die Substantive, Hauptwörter wie auch sich ihnen anordnend Adjektive, Pronomina, Zahlwörter teilt man in verschiedene (8-9) Klassen, die je durch eine besondere Vorsilbe, Präfix geformt verschiedene Wortgruppen bilden. Wir würden am leichtesten diese Konstruktion uns so klar machen:

Schlafen: Mann + schlafen $=$ Schläfer, Leute + schlafen $=$ Schläfer $($ Mehrzahl $)$, Sache + schlafen $=$ Schlafen, Ort + schlafen $=$ Schlafstelle, Leiden + Schlaf $=$ Schlafkrankheit und andere Bildungen.

Außerdem wird im neuen Kongogebiet auch viel die Sprache der Bateke, das Ki-teke gesprochen, ebenfalls eine Kintu, vgl. A. Sims, Vocabulary of the Kiteke (Batio), London 1886, die sich dadurch auffällig macht, daß sie u. a. $t$ in $r$ verwandelt bezw. ausfallen läßt. Da die Bateke ein verhältnismäBig großes Handelsvolk sind, trifft man sie in diesen Gegenden überall. $\mathrm{Da} B$ man daneben noch verschiedene Sprachen und Dialekte (uns sind ja schon vom Fan mehrere bekannt) hört, erscheint nur selbstverständlich, namentlich dringen auch Sprachen vom Norden ein; bei der leichten Erlernbarkeit des Hausa, benutzt man diese Sprache auch häufig als Verständigungsmittel. Auch Fiote hört man in dieser Gegend noch hăufig, da die Fiote, wie bereits gesagt, heute noch $\mathrm{zu}$ Wanderungen gezwungen sind. 


\section{Literatur:}

F a n: Vocabulary of the language, Am. Osorio Zabala, London 1883 in 1895 Actes de la Société phil. tome 24, Paris 1894 bei C. Klincksieck, Seite $1-52$ Grammatik, 53 bis 300 Texte.

Dictionnaire Fan-Français, Lejeune, ebenso Seite $1-52$ dasselbe, 1892 Paris: Quelques principes grammaticaux de la 1 . Fan.

Genesis, London 1894 A. W. Marling u. a.

$\mathrm{M}(\mathrm{Ki}) \mathrm{Pongwe}$ : A grammar of the P. language, Amerik. Miss. 1847, New-York.

Grammaire de la 1. P., Berre, Paris 1873.

Dictionnaire Français-P., Miss. de la Congr. du St. Spirit, Paris 1877.

Dictionnaire Français-P., Gacon, Paris 1881.

P. Gospels, Amerik. Miss. Gabun, New-York 1879 u. m. a.

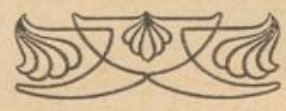




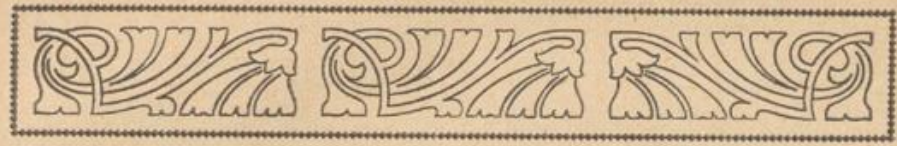

III.

Do000000 ie furchtbarste Krankheit, die keinen verschont,
bleibt das Fieber. Einzelne Gegenden „sind
ganz besonders fiebergesättigt, Fiebernester
im vollen Sinne des Wortes. Daneben kommen
schwere andere Erkrankungen und zahlreiche kleinere Leiden in den Tropen hinzu, wie etwa Leberund Gallenerkrankungen, anämische und skorbutische Erscheinungen, die verschiedenartigen Hautleiden und Infektionskrankheiten, wie Dermatitis (auch roter Hund) tropischer Phagedänismus, Frambösie u. a. m.

4. Gis Diarrhöeische Entleerunge n treten vielfach auf, die zu chronischen Dysenterien ausarten können. Wenn auch der Krankheitsverlauf nicht zu besorgniserregend erscheint, sind Todesfälle daran doch nicht selten. Durch Amöben und Bazillen wird diese Krankheit hervorgerufen. Wer für Durchfall empfänglich ist, muß sich von vornherein größte Ruhe auferlegen, muß sehr genügsam und wählerisch in der Diät sein und darf nie ungekochtes Wasser trinken; auch Salate und Obst sind zu vermeiden, sogar bei der Reinigung von Speisegefäßen darf nur abgekochtes Wasser benutzt werden. Zum Desinfizieren des Wassers habe ich auch mit Erfolg Salzsäure, natürlich nur in ganz geringen Mengen, angewandt.

Rheumatismusartige Zustände mit wassersuchtähnlichen Anschwellungen sind nicht selten, etwa die be- 
kannte Tulatula der Kongoneger. Manche Anschwellungen von Körperteilen entstehen vielfach auch durch Eindringen von Würmern, so die bekannte Elephantiasis durch ungemein kleine Rundwürmer, die Larven der, der Familie der Filariden angehörenden Nematoden. Es entstehen Schwellungen von großem Umfange, namentlich an den Beinen, es können sich Tumoren von 1 Ztr. Gewicht bilden. Im Durchschnitt sind Elephantiasisschwellungen nicht lebensgefährlich nur überaus lästig.

Seltener tritt im Kongogebiet die Beriberikrankheit, Polyneuritis endemica, auf, ein Nervenleiden, dem sicher mangelhafte oder unzweckmäBige Ernährung zu Grunde liegt. Daß fettarme Nahrung wie beispielsweise Reis dieses Leiden begünstigt, kann nicht von der Hand gewiesen werden. Wir könnten also von einer Vergiftung des Körpers durch Nahrungsmittel sprechen, dennoch dürfte eine Infektion auch hier den Anstoß zum Ausbruch der Beriberi geben; sie tritt vielfach unerwartet schnell auf und dehnt sich zu einer Epidemie aus. Lähmungserscheinungen und Schwund sind die hauptsächlichsten Symptome dieses Nervenleidens, doch kann durch Wasseransammlungen auch gegenteiliges Aussehen vorgetäuscht werden. Krämpfe und Zittern sind ständige Begleiterscheinungen.

Ueber den wirklichen Wert von Heilmitteln bei Tropenkrankheiten liegen durchschnittlich noch zu kurze Erfahrungen vor, soda $B$ Martini sagen kann: Ehe ein bindendes Urteil über den Heilwert des einen oder anderen Medikaments gefält werden kann, sind vielleicht jahrelange Beobachtungen sorgfältigster und mühsamster Art nötig. Einstweilen soll aber nicht nachgelassen werden, einerseits weiter nach Heilmitteln zu suchen andererseits die lohnendste Anwendungsweise der bereits versuchten und Aussicht auf Erfolg bietenden herauszufinden. Im Uebrigen bleibt als sonstige 
allgemeine Behandlung eine die Körperkräfte hebende, gute Ernährung durchzuhalten und schlieBlich gegenuber einzelnen schmerzhaften Symptomen je nach Umständen palliativ zu wirken.

Gute Ernăhrung ist entschieden eine der besten Vorbedingungen für dauernde, gute Gesundheit. Daneben werden kuhle Abwaschungen angeraten. Mit dem Baden muß man selbstredend vorsichtig sein. Kurz gesagt, der Gesundheitszustand des Europäers muß geradezu unter Kontrolle gestellt und gehalten werden.

Die größte Gefahr bleibt jedoch das tückische Fieber, die Malaria. Das Wort entstammt romanischen Sprachen, mal-aria, schlechte Luft. Die Malaria tritt überall da auf, wo hohe gleichmäßige Wärme, ein relativ hoher Grad von Feuchtigkeit, das Vorhandensein gewisser Stechmücken (Anophelinen) und das Vorhandensein chronisch malariakranker Menschen sich findet. Jeder Fieberanfall entspricht der Reifung einer Parasitengeneration auf Grund der neuesten Forschungen. Das Fieber selbst tritt in verschiedenen Arten auf, vielleicht auch durch verschiedenartige Infektion bedingt, vornehmlich als „Wechselfieber". Wir nennen die Malaria darum auch Wechselfieber, da das Leiden als ein in bestimmten Intervallen wiederkehrendes Fieberstadium zu betrachten ist.

Daß Parasiten die Schuld an der Malaria zuzuschreiben ist, wurde seit jeher angenommen. Man glaubte, die Malariaparasiten kämen aus dem Boden in den menschlichen Körper (Lufttheorie) oder die Infektion entstände durch Trinkwasser; durch Stoffverfaulen atmete man Miasmen, die Krankheitskeime (Erreger) ein.

Das gelbe Fieber zeigt sich ja auch im Zusammenhange mit den Jahreszeiten und erreicht mit dem starken Auftreten der Stechmücke auch seinen Höhepunkt. Je 
nach Heftigkeit erreicht das Fieber schon nach Stunden oder erst nach Tagen seinen Höhepunkt, Krisis. Anfänglich geringe Anfälle können nach wenigen Tagen doch noch tötlich verlaufen. In Afrika ist die Sterblichkeit daran weit größer als in anderen Lăndern.

Die eigentliche Ursache des Fiebers aber liegt in einer Ansteckung durch die bereits erwähnten Stechmücken. Nicht alle Mosquitoarten eignen sich als Zwischenwirte für die Malariaparasiten (es gibt deren drei) sondern nur die Gattung Anopheles. Wo keine Stechmücken gefunden werden, wird auch kaum Malaria festzustellen sein, und trotzdem fand ich in Paraguay (Südamerika) Anopheles mattogrossensis massenhaft in einer fast malariafreien Gegend. -

5. Der Fieberverlauf von Malaria ist geradezu typisch, er durchläuft Frost, Hitze, Schweiß. Zunächst stellt sich Mattigkeit, Appetitlosigkeit ein, das Fieber steigt $1 / 2-1$ Grad, dann kommt Kältegefühl, Schüttelfrost, es tritt ein bläuliches Anlaufen der Schleimhäute und Fingernägel ein (Cyanose). Der Frost dauert nur wenige Stunden, dann folgt Hitze, die Haut wird heiß, die Lippen trocken, Dauer 4-8 Stunden. Die Temperatur steigt über 40. Hierauf erfolgt Schweißausbruch unter Fallen der Temperatur. Die ganze Dauer beträgt bei leichteren Fieberanfällen 10-12 Stunden.

1) $\mathrm{Da}$ bei jedem Fieberanfall eine größere Zahl roter Blutkörperchen vernichtet wird, so tritt bei den Personen, die sehr vom Fieber heimgesucht werden, eine blutleere Hautfarbe in Erscheinung, sie sehen blaßgelb aus.

Die Malariaparasiten selbst vermehren sich durch ungeschlechtliche Vermehrung, Schizogonie, im Blute und zwar in den roten Blutkörperchen des Malariakranken oder durch geschlechtliche Vermehrung, Sporogonie, im Körper der Stechmücke. Die Parasiten selbst teilen fast alle Malariaforscher in 3 Spezies: 
1. Tertianaparasiten mit 48 stündiger Entwicklungsdauer

2. Quartanaparasiten mit 72 stündiger Entwicklungsdauer

3. Pernitiosaparasiten, nach Koch Tropicaparasiten.

Während die Tertiana und die Tropica nur auf die Tropen beschränkt sind, geht die Quartana mehr polwärts, in Europa etwa bis Süd-Schweden.

Das einzige Heilmittel, obwohl man von einem Heilmittel im unmittelbaren Sinne natürlich nicht sprechen kann, ist bis heute das Chinin geblieben. Man benutzt die von der Chinarinde gewonnenen Präparate $\mathrm{Chi}$ $\mathrm{n}$ in um hydrochloricum und bisulfuricum, also die leicht im Wasser löslichen Salze des Alkaloids. Am geeignetsten empfehlen sich $1 / 2$ Gramm enthaltende Tabletten oder Chinin in Oblaten, da das Chinin in wässeriger Lösung überaus ungern genommen wird. Besonders angenehm sind Chininschokoladenpastillen, in denen $1 / 2$ Gramm Chinin gelöst ist. Sie wurden von mir in den Tropen mit gutem Erfolg benutzt. Hergestellt werden dieselben wohl von einer Fabrik im Rheinland.

Das Chinin wirkt tötend oder doch schädigend auf die Malariaparasiten, man muß es jedoch möglichst schon einige Stunden vor dem zu erwartenden Anfall einnehmen. Die Dosis an Chinin und ihre Ordination richtet sich nach dem Arzte. Mehr wie ein Gramm wird für den Anfang, bei täglicher Verabreichung vielfach nicht vertragen. Jedenfalls kann man sich durch $1 / 1$ gr. Gaben vor der Ankunft in fieberreichen Gegenden teilweise schützen, doch nie immunisieren. Man muß das Chinin aber am Körper ausprobieren, denn manche Personen leiden unter seiner Wirkung, sie bekommen Herzklopfen, Ohnmacht, Erbrechen u. a. Wer eine Ausreise nach Deutsch-Kongo unternimmt, muß sich jedenfalls unter allen Umständen auf Tropentaug-

Krü ger, Das neue Kongogebiet. 3 
lichkeit von einem Arzte untersuchen lassen, wozu das Ausproben der Chininwirkung ebenfalls gehören sollte.

Obwohl Chinin sehr unangenehm schmeckt, ist es noch immer das beste "Antifebrin" für Fieber, mit Geschmackskorrigentien „lohnt" es nicht; wo man Oblaten nicht zur Hand hat, hilft man sich in der „Wildnis“, indem man das Medikament in Zigarettenpapier einwickelt und mit einem Schluck Wasser oder Wein hinunterwürgt.

Gerade der schlechte Geschmack des Chinins ist es, der die größte Unterlassungssünde, es regelmäßig zu nehmen, zeitigt.

Eine weitere Fieberart, vielmehr eine Folgekrankheit der Malaria ist Febris bilosa haemoglobinurica, das Schwarzwasserfieber, das dadurch entsteht, daß das Hämoglobin nicht mehr in Gallenfarbstoff umgesetzt wird, sondern durch die Nieren im blutig gefärbten Urin ausscheidet. In starken Fiebernestern findet sich auch mehr oder weniger das Vorkommen von Schwarzwasserfieber. Der Urin erscheint braunrot bis schwarz(rot). Der Blutzerfall kann ein so großer sein, daB an einem Tage weit über eine Million roter Blutkörper zerstört werden. Daß bei einer starken Nierenarbeit die Kanäle sich verstopfen und daher Anurie (Harnverhaltung) eintritt, erscheint nur selbstverständlich; hierdurch erfolgt meist auch der Tod, ebenso wie durch allgemeine Schwäche.

Das ausgesprochene amerikanische Gelbfieber, das auch nach Afrika verschleppt wurde, kommt hier jedoch nur wenig vor; es beschränkt sich größtenteils auf die Küstenbezirke. Um so unheilvoller bleibt das Malariafieber; über Ursachen, Verlauf, Verhütung und Heilfaktoren, sowie über die Schlafkrankheit erscheint von mir im gleichen Verlag ein Buch: Das Tropenfieber und die Schlafkrankheit (Malaria und Trypanosomen) für Reisende, Ansiedler und Kolonialinteressenten 
postfrei für $1,50 \mathrm{Mk}$. zu beziehen. Ich lege darin Wert auf Darstellung und Beobachtung für Laien, da diesen in Europa nur wenig, in den Tropen überhaupt keine Literatur zu Gebote steht.

Daß man nun mit allen Mitteln gegen das Ausbreiten der Malaria vorgeht, ist natürlich. Hauptsächlich trachtet man nach einer Verminderung der Bodenfeuchtigkeit, Drainage und Bodenkultur. Dazu gehört auch eine Bepflanzung mit stark wasserentziehenden Gewächsen. Als solcher kommt etwa der Fieberbaum Eucalyptus globulus in Betracht, der dem Boden schnell die Feuchtigkeit entnimmt und ihn austrocknet.

Immerhin ist ja der Untergrund mit organischen Stoffen dort überladen, wo Malaria vorkommt. Als erste Bedingung bleibt daher nötig, der Malariamücke den Boden zu entziehen. Der bedeutende Tropenarzt Ziemann sagt in seinen Malariaarbeiten: Es ist gar keine Frage mehr, daß die weiße Rasse, wo ihr die Besiegung der Malaria gelingt, und wo sie sich frei hält von der Vermischung mit minderwertigen, farbigen Rassen, Akklimatisation in den Tropen erreichen kann; nur tropische Tiefländer mit hoher Feuchtigkeit der Luft und sehr geringen monatlichen und täglichen Schwankungen der Temperatur müßten ausgeschlossen sein.

Eine drohende Gefahr bildet dann aber auch noch ein weiteres Leiden für den Ansiedler, die Schlafkrankheit. Die Verbreitung der Trypanosomenkrankheit, die schon über hundert Jahre bekannt ist, beschränkt sich in Afrika auf die zentralen Fluß- und Seeniederungen; sie nistet sich besonders unter der armen, notleidenden Negerbevölkerung ein. Die menschliche Trypanosomenkrankheit tritt als Schlafkrankheit auf, die Benennung zeigt uns schon das hauptsächlichste Symptom. Sie verläuft meist chronisch und wird hervorgerufen durch einen Erreger, Parasiten, der seinerseits wieder durch den $\mathrm{BiB}$ einer bestimmten 
Tsetsefliege, Glossina palpalis auf den Menschen übertragen wird. Die Trypanosomen werden vornehmlich in der Rückenmarkflüssigkeit, den Lymphdrüsen und dem Blute festgestellt. In Deutschkongo sind fast alle Flußgebiete vollständig verseucht und man geht nicht zuweit zu sagen, daß auch dort die Schlafkrankheit noch im Zunehmen begriffen ist. Am bedauerlichsten ist es, daß es heute noch unmöglich ist, die Trypanosomenkrankheit zu heilen, ja, daß alle therapeutischen Maßnahmen geradezu aussichtslos sind. Mit teilweisem Erfolg wurde Arsenik, daneben Jod angewendet. Man hat um so weniger Wirkung, als ein Hauptsymptom, der große Kräfteverfall, eben durch das appetitlose, schlafende und (indirekt) gleichgiltige Verhalten des Patienten nicht aufgehalten wird, wollte man auch jede künstliche Nahrungszufuhr ermöglichen.

Aber nicht nur die eine Tsetse dürfte in Frage kommen, sondern auch wohl noch andere Glossinenarten; die Palpalis unterscheidet sich von anderen durch eine besonders dunkle Farbe.

Es ist richtig, daß in Französisch-Aequatorialafrika ebenso wie im belgischen Kongostaat die Schlafkrankheit große Verbreitung gefunden hat, das gibt die deutsche Regierung selbst zu; dies wäre wahrscheinlich nicht in dem Umfange geschehen, wenn die Franzosen rechtzeitig die nötigen Maßnahmen gegen sie ergriffen hätten, wie wir es in Kamerun getan haben, sobald die Krankheit dort festgestellt wurde. Wenn erst eine systematische Bekämpfung einsetzt, ist auf Grund der verhältnismäßig guten Erfolge, die wir in Ostafrika und vor allem in Togo erzielt haben, anzunehmen, daß die Gefahr überwunden wird. Erleichtert wird uns dies dadurch, daß wir den Herd der Schlafkrankheit am Sanga direkt angreifen können Die Bekämpfung der Schlafkrankheit im bisherigen Gebiet von Kamerun dürfte hierdurch wesentlich vereinfacht werden. Besonderen Erfolg wird 
man sich von einem Zusammenarbeiten der interessierten Măchte versprechen können, wie es zwischen Deutschland und England sowohl für Ostafrika wie für Togo durch besondere Abkommen gewăhrleistet ist. Es ist nicht daran zu zweifeln, daß auch Frankreich bereit sein wird, mit uns hierin Hand in Hand zu arbeiten.

Aehnlich wie Malariaparasiten entwickeln sich auch im Körper der Tsetsefliege die Trypanosomen. Robert Koch sagt darüber: Im Verdauungskanal der infizierten Glossinen wachsen die Trypanosomen zu erheblicher Größe heran und lassen dann zwei ganz verschiedene Typen erkennen. Der eine (weibliche) ist charakteristisch durch starken Breitendurchmesser, reichlichen Gehalt an Plasma, welches bei Anwendung einer Färbung einen blauen Farbenton annimmt, und einen rundlichen Kern von lockerm Gefüge. Der zweite (männliche) Typus zeigt einen geringen Durchmesser und infolgedessen eine schlanke Gestalt, vollständigen Mangel an blaufärbbarem Plasma und einen langgestreckten fast stabförmigen Kern von dichtem Gefüge, welche eine dunkle gleichmäßige Chromatinfärbung annimmt.

Bis zum Ausbruch der Schlafkrankheit können nach stattgehabter Infektion Monate, ja Jahre vorübergehen. Zunächst tritt dann aber Neigung zu Schläfrigkeit auf, schließlich reagiert der Kranke noch nicht einmal auf Anruf oder AnstoB. $\mathrm{Er}$ taumelt trunken umher, Schlafsucht tritt ein, doch sind die Sinnestätigkeiten noch erhalten. Meist schwellen auch die Nackendrüsen an, ohne jedoch zu vereitern. Die eigentliche Krankheit dauert mehrere Monate, doch zieht sie sich auch Jahre hindurch, kann auch mit der 3. Woche schon ihr Maximum erreicht haben. Es richtet sich hierbei natürlich wieder ganz nach der Körperbeschaffenheit jedes einzelnen Infizierten.

Zur gründlichen Beseitigung der Schlafkrankheit gehören bedeutende Kosten und dennoch eine völlige 
Ausrottung ist uns undenkbar, wenn die angrenzenden Gebiete nicht auch ebenso große Anstrengungen zur Eindämmung dieser fürchterlichen Krankheit in die Wege leiten. Dies aber erscheint auf Jahre hinaus noch ziemlich ausgeschlossen, - da vor allem in französischen Werken über die Schlafkrankheit sich mehrfache Hinweise finden, daß dieselbe jetzt noch typisch bleiben wird, ehe nicht eine völlige Regulierung der Verhältnisse ermöglicht werden kann. Dies aber ist auch nicht in einem Menschenalter geschehen, wenigstens nicht von seiten angrenzender Staaten.

Man wird gegen die Schlafkrankheit, wie gegen das Fieber noch manche Neuerung erwarten können. Viele Forscher, erste Kapazitäten studieren und arbeiten an dem Problem. Erst kürzlich haben wir wieder etwas von einem Arzt darüber gehört. Dr. Mehnarto, ein Mitarbeiter Robert Kochs, ist von einer Studienreise zur Untersuchung der Schlafkrankheit auf den Inseln des Viktoria-Niansa vom Kongo hier eingetroffen. Dr. Mehnarto hat ein verläßliches Mittel gegen alle Trypanosomakrankheiten entdeckt und diese selbst erprobt, nachdem er die Schlafkrankheit eingeimpft hatte, die eine sechstägige Bewußtlosigkeit verursachte. Die Entdeckung macht ein ungeheures Aufsehen. Und wenn wir auch keine vorzeitigen Loblieder singen wollen, so wissen wir doch unbedingt, da $B$ an der Ausrottung der Tropenkrankheiten gearbeitet wird. Kleine Erfolge zusammengetragen, werden doch endlich einmal einen Monumentalbau ergeben.

Die medikamentöse Behandlung hat eigentlich bisher immer versagt. Am meisten wird mit Arsen gearbeitet und kommt ihm vielleicht die einzige Wirkung noch zu. Ein verhältnismäBig weniger giftiges Arsenpräparat zur Behandlung (Abtöten) der Trypanosomen ist Meta-Arsensäureanilid, Atoxyl, ein weißes, in Wasser leicht lösliches Mittel. Man spritzt das Atoxyl in etwa 
$10 \%$ Lösung (Tagesdosis davon bis $0,2 \mathrm{~g}$ ) unter die Haut; wie gesagt, ist auch dieses Mittel ein arsenhaltiges $(38 \%$ Arsenik) und daher nur mit Vorsicht anzuwenden, auch ist die subkutane Injektion damit sehr schmerzhaft.

Unwirksam zeigte sich das Trypanrot, ein der Benzoepurpurreihe angehörigerFarbstoff, ebenso Brillantgrün, das Sulfat des Tetraäthyldiparaamidotriphenylcarbidrid, noch unwirksamer Malachitgrin, Benzidin, Chrysoidin u. a. Von der Safrantherapie aber will ich ganz absehen.

Als Prophylaxe kämen zwei Wege in Betracht, der eine müßte auf einen Speisegenuß hinweisen, der einen Geruch entstehen ließe, wodurch die Tsetsen den Menschen meiden, der andere müßte den Inhalt der GefäBbahnen gegen Trypanosomen geradezu immunisieren. Letzteres hat hin und wieder Wirkung durch den Genuß arsenikhaltiger Mittel, etwa der sogen. asiatischen Pillen, doch wird die Gefahr der Ansteckung dadurch doch nicht mit Sicherheit beseitigt.

Die Beseitigung der Tsetsen und damit der Trypanosomen bedarf der gröBten Beachtung. Eine neue Methode zur Bekämpfung der Schlafkrankheit wird wieder gemeldet. Ein Plantagenleiter auf der Insel Principe, die ja unserem Kongogebiet vorgelagert ist, hat ein neues Mittel zur Bekämpfung der Schlafkrankheit gefunden. Er bemerkte, daß die Glossina auf die Rücken der Arbeiter vornehmlich dann zuflogen, wenn diese bei der Hackarbeit, also in gebückter Stellung, beschäftigt waren. $\mathrm{Er}$ gab nun diesen Arbeitern ein schwarzes Gewand auf den Rücken, das auf der Außenseite mit einer klebrigen Substanz bestrichen war. Die portugiesische Kommission, die zur Erforschung der Schlafkrankheit sich auf der Insel befand, bat oft den Ansiedler, Leute mit schwarzen Gewändern dorthin zu schicken, wo eine große Anzahl schwarzer Fliegen ge- 
sehen worden war. Gewöhnlich waren zwei Leute genug, um diese Plätze in kurzer Zeit gangbar zu machen.

Ein Plantagenbesitzer in Ostafrika hat die Methode aufgenommen und einige Versuche mit Lorantus ficusund Euphorbien-Leim angestellt, die ausgezeichnete Resultate lieferten. Nach den Aussagen eines Missionars sollen schon früher Neger am Lopori-Fluß im Kongostaat sich mit einer kosmetischen Salbe beschmiert haben, die Glossinen abschreckt. Die Wangombe, die diese Salbe benutzen, blieben von der Schlafkrankheit verschont, während ihre Nachbarn, die sich ihrer nicht bedienten, dahinstarben.

Wir haben auch einige Pflanzen, die die Fliegen, selbst die großen Stallfliegen verscheuchen und Versuche mit solchen Pflanzen, zu denen in erster Linie auch die Petersilie gehört, wären angebracht. Verhindert man die Ausbreitung der Tsetsen, wird man auch die Schlafkrankheit erst ausrotten können. Heute haben freilich Neger wie Weiße, Arbeiter wie Ansiedler darunter zu leiden. $\mathrm{Da \beta}$ mehr Eingeborene davon befallen werden, liegt doch nur an der weit größeren Anzahl, der nur sehr wenige Europäer gegenüber stehen.

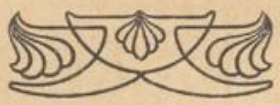




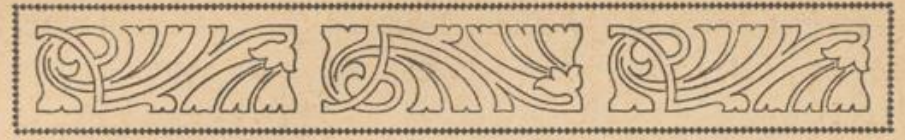

IV.

000000000 n letzter Zeit ist verschiedentlich die Ansiedlungsmöglichkeit im Deutschkongogebiet ausgesprochen worden. Die Möglichkeit kann von energischen Männern, von 00000000 denen sich freilich jeder als ein Stanley fühlen
sollte, zur Tat werden. Freilich stellen sich auch für den einzelnen oder für einzelne große Gefahren entgegen, dennoch sollte man bedenken, daß auf dem Kongo ein reges Leben herrscht. Was ist aus dem Flußgebiet seit 1855 , als zuerst an der Kongomündung eine französische Faktorei angelegt wurde, geworden? $\mathrm{Da}$ herrscht Leben und Tätigkeit. Große Kapitalien sind dort investiert, die bedeutende Arbeit leisten, aber auch mit großem Verdienst abschließen. Von diesem Sinne aus kann man die beiden Zipfel, die Deutschland jetzt an den Kongo und den Ubangi führen, nur begrüßen. Was haben die Nieuwe Afrikaansche HandelsWennootschap zu Rotterdam oder die British Congo Company zu Manchester, die aus der portugiesischen Central Africain Trade Company zu Lissabon hervorgegangen ist, an Waren nach Europa geschleppt. Großzügigkeit und Intelligenz freilich regieren jedes überseeische Unternehmen. Manche deutsche Kolonialgründung mußte an der Kleinlichkeit der europäischen Geldinteressen scheitern. Jedenfalls schafft das neue Kongogebiet tatkräftige nützliche Arbeit und der Lohn 
ist bei umsichtiger Leitung sicherer als ein Arbeiten im Hinterlande Kameruns ohne Bahnen und ohne Zugang zum Kongo und zum Ubangi.

Eine völlige Akklimatisation der weißen Ansiedler und Händler ist kaum zu fünf Prozent möglich; die Bedingungen dazu müßten erst im Laufe der Zeit gebessert werden.

Das Hauptaugenmerk muß heute auf die Ansiedlungen im allgemeinen gelegt werden. Die Anlage von Plantagen im neuen Gebiet erfordert viel Umsicht. An die wogende Grassteppe mit schmutziggelber Färbung wird die künftige Wohnstätte des Kolonisten gelegt. Aus Stielen der Raphia vinifera wurden kleine Gebäude hergestellt, ehe mit einem geräumigen, entsprechenden Haus begonnen wurde. Heute richtet man sich auf Zelte, Hausbauten und Geräte möglichst schon in Europa ein.

Wo Plantagen angelegt werden können, zeigen uns zunächst die Wasserverhältnisse. Es muß gutes Trinkwasser vorhanden sein, aber auch die Nähe eines möglichst befahrbaren Flusses. $\mathrm{Zu}$ große Feuchtigkeit, also Sumpfgebiet muß hingegen unter allen Umständen gemieden werden. Dagegen muß man natürlich auch mit etwaigen Ueberschwemmungen zur Regenzeit rechnen.

Der Baugrund soll fest und wenn möglich felsig sein, auf einem Hügel, jedenfalls so hoch wie nur irgend möglich liegen. Beim Hausbau achtet man auf einen guten, sicher abschließenden Boden, auch auf Schutz vor Sonnenstrahlen. Auf die Gefahren der Hitze muB man selbstredend sehr bedacht sein. Sonnenstich und Hitzschlag müssen von vornherein vermieden werden, selbst nach leichten Anfällen bleiben dauernde nervöse Störungen zurück. Trinkwasser findet sich nur selten vorzüglich, man muß alles Wasser sorgfältig filtrieren und es empfiehlt sich außerdem, es zuvor noch abzukochen. Unterläßt man das, so werden sich früher 
oder später dadurch Gesundheitsschädigungen einstellen.

Ansiedlungen im neuen Kongogebiet sind jedenfalls mit vielen Schwierigkeiten verbunden, wie die kurze "Geschichte“ dieser Kolonie uns schon lehrt. Die wenigen französischen Militärposten kamen kaum über ihre Ansiedlungen hinaus, da mit den Eingeborenen schwer Kirschenessen und ihnen nicht zu trauen ist. Außerdem wechselt die Besatzung so häufig, daß ein Nutzen für die ganze Kolonie unmöglich wird.

Wir glauben sicher, daß manche Kolonisten, die zum neuen Koloniallande auszuwandern gedenken, wenn sie über den Kongo vordringen würden, noch im Kongostaat sitzen bleiben werden. Am Kongo entlang finden sich viele Ansiedlungen, die freilich heute sicher dem Neuling mehr bieten als das deutsche Kongogebiet.

Keinesfalls darf der Ansiedler von Geld entblößt sein, wenn er sich in dem neuen Kongogebiet seßhaft machen oder nur Handel treiben will. Wir müssen deutsches Kapital zu interessieren suchen, können aber vielleicht prognostizieren, daß erst manche Millionen vergebens geopfert werden müssen. Kaum eine Pflanzungsgesellschaft wird anfangs günstig abschließen, wenn auch die Einladungen zur Zeichnung anders erscheinen mögen. Eine Müdigkeit in Kolonialwerten darf nicht noch gesteigert werden; das wollen auch wir nicht. Aber mit Umsicht wird manche Gesellschaft nach längerer oder kürzerer Zeit prospirieren, dabei möchten wir bemerken, daß durchschnittlich Privatunternehmungen besser arbeiten, nicht zuletzt dadurch, daß ihre Direktion freie Hand zu rechtzeitigen Erfolgen hat, eine Gesellschaftsleitung aber in fast allen Fällen erst noch von dem grünen Tisch und seinen vielfach zaudernden Mitgliedern abhängig sein wird.

Die gröBte Notwendigkeit wird darin liegen, Wege 
anzulegen und zu unterhalten, Verbindungswege für den Handel (sagt schon Cameron). Zur Anlage von Straßen stellen sich keine nennenswerten Terrainschwierigkeiten in den Weg. Aber nicht nur Kommunikationen anzulegen ist nötig, sondern die Straßen müssen auch unterhalten werden. Was heute geschaffen wurde, kann morgen schon wieder von Rasen bedeckt, von Schlingpflanzen überwachsen sein.

Menschenkraft wird noch lange das sicherste Transportmittel bleiben, wenn auch sicherlich ein teueres. Träger wird es noch lange geben, bis sich diese "Zunft" in Dienstleute verwandeln wird. Nichts destoweniger ist eine Trägerkarawane nur ein äußerst primitives Transportmittel, wenn die einzelnen Träger auch sehr ausdauernd und kräftig genannt werden müssen. Mit der Einführung von Reitstieren wäre auch nur wenig gedient, da die Rinder nur kurze Zeit, wie es sich bestätigt hat, diesen Dienst zu verrichten imstande sind und mithin zu teuer werden.

Auch andere Arbeitsmittel werden schwer zu beschaffen sein. Selbst die Elefanten können Sumpfgegenden auch nur unter Schwierigkeiten passieren, ganz abgesehen, da $\beta$ der afrikanische Elefant, heute wenigstens noch, als ziemlich unzähmbar gilt.

Da man also auf Karawanen angewiesen ist, wird man auf ihre Zusammenstellung große Sorgfalt zu verwenden haben. Man wird die Träger- und Begleiterzahl tunlichst vermindern. Karawanen zusammenstellen aber ist eine überaus schwierige Arbeit. Da muß man mit der Individualität jedes einzelnen rechnen. Bei den Karawanen sollen in besseren Stellungen, nicht zuletzt etwa als Dolmetscher, nur gewissenhafte und junge Neger Verwendung finden. Alte scheide man unbedingt aus, sie eignen sich weitmehr auf den Ansiedlungen als Aufseher. Bei engagierten Trägern hat man die Namen zu notieren, aber auch den Namen ihrer Häupt- 
linge. Bei Desertionen und Streitigkeiten kann man nur so auf Beilegung und Erfolg rechnen.

Die Ausrüstung will überlegt werden, sie richtet sich selbstverständlich nach dem Zwecke und dem Ziele des Reisenden. Jedes übergroße Gepäck muß möglichst vermieden werden. Eine Zusammenstellung anzugeben, wäre zwecklos, da Ansprüche und Geldmittel außerdem ganz verschieden sind. Man nimmt Kisten und Blechkasten als Verpackungsmaterial, vielfach genügen auch Flechtereien aus Binsen und Campinenhalmen.

Große Reichtümer sind zu holen, wenn Mittel und Wege geschaffen werden (sagt Müller), nun wir dürfen nicht zu optimistisch sein, wollen es auch nicht. Erst die Zukunft kann uns lehren, ob auch jeder einzelne Kaufmann und Kolonist auf seine Rechnung kommt.

Die Neuerwerbung bleibt eben ein Wechsel auf die Zukunft, besonders wenn man den Kleinunternehmer hervorheben will. Aber Aussichten und nicht die schlechtesten sind für Deutschkongo entschieden vorhanden, vor allem auch da die Regierung den Ansiedlern in möglichstem Entgegenkommen helfen wird. Bedenken, - wie wir sie heute gegen dieses Neuland für das Deutsche Reich allenthalben hören, - sind früher bei jeder einzelnen kolonialen Neuerwerbung geltend gemacht und dennoch haben wir, obwohl wir im Gegensatz zu anderen Staaten weit mehr Kapitalien geopferthaben, schon heute durchgängig eine gute Nutzung Und daß wir einen Zugang zu den beiden Flüssen haben, wollen wir als unbedingt Notwendiges ansehen. Auf einen Zugang zum Kongo und Ubangi ist von den kolonialen Kennern immer wieder Wert gelegt worden. Ebenso wurde Wert darauf gelegt, beide Ufer des schiffbaren Sanga im Interesse des Verkehrs und Handels zu erhalten. An den Ubangi heranzukommen, an einen Ort, wo der Fluß noch schiffbar ist, war eben- 
falls von Wichtigkeit. Dies ist gelungen. Die Franzosen benutzen den Fluß zum Transport von Truppen, Proviant, Waren nach dem Nordgebiet. Das werden wir nunmehr auch tun können.

Vorzügliche Wege zu bauen, ist möglich, ungeheure Reichtümer wären dann zu holen, eine Eisenbahn ist Lebenszweck (sagt Grenfell) oder der Nutzen, den der Handel im Kongogebiet zieht, wird immens sein, sobald Bahnen gebaut sein werden (François). Eisenbahnen haben alle Reisenden befürwortet, wenn auch Chavanne gegen ein Stanleysches Unternehmen wettert. Eisenbahnen werden als die Quintessenz der Erschließung angesehen, nur fragt es sich, ob sie sich auch rentieren werden.

Mit der Eisenbahn ist die Lebensfähigkeit des Handels im Kongogebiet voll gesichert, sagte Wißmann. Und auch der neue Kolonialbezirk wird durch Verbindungswege und Eisenbahnen sicher gefördert. Aber auszusprechen, daß sich in kurzer Zeit schon eine Eisenbahn rentieren wird, halten wir entschieden für unmöglich oder doch für verfrüht.

Die Ansiedler kämpfen selbstredend für gute Verbindungen, sie haben solche nötig, doch sonst hört man von den Handeltreibenden wenig, damit würden sie sich schädigen und nur die Konkurrenz in ihr Gebiet locken (Stanley).

Jetzt kommt es weiter auf die Erwerbsaussichten an, wieweit sich Kommunikationen verlohnen werden. Die Anbauten und Kulturen von Pflanzen stehen natürlich hier in der ersten Linie. Viele Pflanzen sind ohne Nachhilfe leicht anzubauen. Dagegen könnte die Unrentabilität von großen Gesellschaften angeführt werden.

Da einzelne Konzessionsgesellschaften die ihnen von der Regierung auferlegten vielen Verpflichtungen natürlich nicht einhalten können, sie sind wirklich augenblicklich noch nicht dazu in der Lage, bleibt ihnen 
nichts anderes übrig als zu liquidieren und ihre so schon mühsam „beieinander gehaltene" Gesellschaft sang- und klanglos aufzulösen.

Chavanne dagegen lehrt uns: Die Zukunft des Handels im Kongogebiet ist eng an die kulturelle Bewirtschaftung des Bodens gebunden, und diese wird auch den Entwickelungsgang des Handels bestimmen. Zur Stunde, wo niemand dem Gebiet die Untauglichkeit $\mathrm{zu}$ intensiver Bodenkultur nachweisen kann, da dasselbe kaum in den dürftigsten Linien erforscht und auch nirgends noch der Versuch seines Anbaues tropischer Nährstoffe und Genußmittel gemacht würde, mußte jedes voreilige Urteil ein irriges sein, sicher läßt sich nur der Satz aufstellen, daß Bodenkultur die Basis jedes gedeihlichen Aufschwunges des Handels im Kongogebiete sei.

Wir veranlassen niemand $\mathrm{zu}$ kühnen Illusionen über die Produktionsfähigkeit des neuen Gebietes, aber daß die Vegetation dem Handel keine nennenswerten Produkte liefert, halten wir für ausgeschlossen. Und selbst, wo heute die Beackerung kleiner Gebiete nur von Negern vorgenommen wird, genügt sie schon $\mathrm{zu}$ deren Lebensunterhalt. Und doch arbeitet landwirtschaftlich nur das Weib beim Neger. Die Frauen besorgen die Ackerbestellung, sie bauen gerade das, was zum Lebensunterhalt sich nötig macht, freilich oft mit primitiven Geräten. Der Weiße muß sich selbstredend schwerer Feldarbeit enthalten und in der Sonne darf er körperlich überhaupt nicht arbeiten. Aber zu aussichtslos halten wir das erworbene Gebiet nicht.

Leon Guiral spricht schon in Congo français du Gabun à Brazzaville, Paris 1889, dem neuen deutschen Ġebiet eine große Zukunft zu, wir können uns seinen Ansichten auch anschließen, wenn wir freilich heute noch das Deutsche Kongoland als ein "Wechsel auf die Zukunft" ansprechen müssen, es ist keineswegs 

leicht ohne genïgende Geldmittel festen FuB zu
fassen.

Nun zu einigen Kulturpflanzen. Da ist zunächst Maniok, Jatropha manihot (Euphorbiacee), roh für Menschen freilich giftig, durch Kochen und Rösten macht man sie jedoch sehr schmackhaft. Die Knollen werden abgeschält und in der Glut geröstet, sind sehr gut im Geschmack oder man trocknet Stücke an der Luft, zerschlägt und zerstößt sie zu Mehl. Die Knolle ist rein weiß, und ihr Geschmack will erst "erlernt" sein. Die Neger haben noch ein besonderes Tafelrezept für diese Frucht, Maniok wird nämlich zu Kugeln geformt, in Palmöl getaucht und in den Mund geschleudert. Maniok ist eigentlich eine Giftpflanze, die in Mittelamerika beheimatet ist und deren Wurzeln mit reichem Gehalt an Stärkemehl bis $1 \mathrm{~m}$ Länge und $40 \mathrm{~cm}$ Breite erreichen können. An Gift enthalten die Wurzeln Blausäure und müssen vor dem Genub zum mindesten am Feuer in Tongefäßen oder auf Eisenplatten gut getrocknet werden.

Andere zur Kultur wichtige Pflanzen gedeihen überall, so die Paradiesfrucht Banane, Mais und Hirse, und die aus Amerika stammenden Bataten, Convulvulus batatus; ihre mehlreichen Wurzeln werden wie Kartoffeln verwendet, haben jedoch einen süßlichen Geschmack. Man muß jedoch in der Ernährung auf Abwechslung sehen, da man sich sonst leicht Erkrankungen zuzieht. Viel Bananenessen etwa erzeugt eine hartnäckige Stomatitis, die sich auch in Parotisschwellung (Ziegenpeter) kundgibt.

Die ErdnuB Arachis hypogaea, den Eingeborenen ein beliebtes Nahrungsmittel, gesotten oder geröstet wird sie genossen, ist ja auch bei uns allgemein bekannt. Das Sammeln von Erdnüssen ist eine Hauptbeschäftigung der Neger. Die Nüsse, sog. Kameruner werden mit $20 \mathrm{Mk}$. der Zentner in Hamburg gehandelt, 48 
bringen also einen ganz ansehnlichen Gewinn. Außerdem liefert die Erdnuß ein brennbares Oel.

Es wächst auch Sterculia (Cola) acuminata, die KolanuB aus der Familie der Stinkbäume hier wild, die einen hohen Coffeïngehalt besitzt. Ueberall wird Kola gekaut, vor allem ihr durch Gerbsäuregehalt eine Vorbeuge vor Ruhr zuzuschreiben ist. Sie "wirkt“ durch starke Herztätigkeit, Nervenreiz und Erhöhung der Munterkeit. Die Nüsse werden von manchen Negern den ganzen Tag über gekaut und der sich bildende Speichel verschluckt.

Die Basis aller Geschäfte bildet neben der Erdnuß die Oelpalme mit ihrem Palmöl und Palmkernen. Elaeis wird $10-15 \mathrm{~m}$ hoch. Die Bäume tragen $20-25$ Stück 6-7 m lange Wedel. Die Früchte stehen in Fruchtständen und bestehen aus zahlreichen Einzelfrüchten. Die Früchte werden pflaumengroß, fettglänzend dunkelgelb bis rot. Das fetthaltige Fruchtfleisch und die Kerne werden als Viehfutter oder zur Verarbeitung auf Oelkuchen für das Vieh verwendet.

Palmöl, namentlich aus der Guineaelaeis wird in dortigen Gegenden in reicher Menge gewonnen und dieser Gewinn kann durch Kultur der Oelpalme noch gesteigert werden. Das veilchenartig schmeckende, dunkelrote Palmöl bildet einen wichtigen Handelsartikel. Der reichliche Saft wird gekocht, nachdem die Palmnüsse zuvor zerkleinert und gestoßen wurden.

Ein guter Handelsartikel verspricht auch Kopal zu werden; es gibt Kopalharz, ein teures Hartharz in gelben und weißen Qualitäten als Sekretion verschiedener Mimosen, daneben wird sich viel fossil (afrikanischer Bernstein) finden, doch meist fetisch, d. h. es durfte bisher nicht berührt werden und die Fundstellen hielt die Bevölkerung streng geheim.

Der Wert von fossilem, durchsichtigen, weißen und roten Kopal (Kopalgummi) ist bekannt. Viel Kopal 
wird zu Fackeln von den Eingeborenen verbraucht. Man trifft den fossilen Kopal oft in großen Fladen, selbst bis zu einem halben Meter Durchmesser.

Vielleicht gibt es auch Wachs und Honig einzutauschen; der Honig soll in manchen Gegenden von herrlichem Aroma sein. Das Wachs wird in Binsen verpackt und mit Lehm verschmiert, einzelne Stücke durchgängig von Zentnerschwere.

Ein Haupthandelsartikel bleibt dagegen der Kautschuk. Die verschiedenen Gummipflanzen liefern zwar nicht gleichguten Kautschuk. Schon Stanley sprach die Meinung aus, daß die Kautschuklieferung eines Jahres hinreichen würde, die Kosten einer Kongoeisenbahn zu bestreiten.

Der erste Kautschuk wurde 1867 geliefert, seitdem treibt man Raubbau. Am elastischen, aber zähen Gewebe der Liane rinnt nach Einschnitten eine rosigweiße Milch, Latex, stundenlang, reichlicher in der Regen-, als in der Trockenzeit. Erst ist der Saft schneeweiß, dann wird er jedoch dunkelgrau (im Innern bleibt er noch nach Monaten weiß). Am besten kommt die Kautschukpflanze, z. B. Landolphia, im Schatten der Bäume fort. Ihre weißen Blüten mit Orangenduft stehen doldenartig zusammen; die Früchte gleichen Apfelsinèn, haben große Kerne und ein säuerlich schmeckendes Fruchtfleisch.

Ein weiteres wichtiges Handelsprodukt ist das Elfenbein. Elfenbein wird in zwei Arten "grün" und "tot" gehandelt, ersteres stammt von erlegten Elefanten, letzteres findet man hin und wieder bei verendeten Tieren; ersteres, das teuere, ist weiß oder gelblich, letzteres braun. Es wird nach Gewicht gehandelt; jeder Zahn wiegt zwischen 10-20 Kilo. Das teuerste Elfenbein ist das grüne aus Gabun und Deutsch-Kongo.

Ferner ist der Tierhandel kein schlechtes Geschäft, nur wird er sich als selbständige Erwerbsquelle 50 
nicht rentieren. Junge Schimpansen werden an der Küste mit $150 \mathrm{Mk}$. gehandelt, jedoch sterben viele Tiere, da sie Gefangenschaft und Klimawechsel schwer vertragen können.

Die Anwohner von dem Deutschkongogebiet, das südlich an den Kongo trifft, nördlich von Lukolda, züchten sogar Krokodile, wie uns schon Stanley, der Bula-Matari (Felsenbrecher) berichtet; mit der Züchtung von Krokodilen treiben sie eine gewinnbringende $\mathrm{Be}-$ schäftigung. Die Häute von Krokodilen werden gut bezahlt, sonst aber ist die Nachfrage nach Tierhäuten nicht sonderlich grob.

Auch Sammlungen lassen sich anlegen und $\mathrm{zu}$ Gelde machen. Im üppigen Pflanzenwuchs tummeln sich beispielsweise die farbenprächtigsten Schmetterlinge und Käfer finden sich ebenfalls um und an den Bäumen. Dieses Sammeln und Zubereiten freilich will erlernt sein, genau wie das Abbalgen von Vögeln und Säugetieren.

SchlieBlich sind auch Mineralerze sicher abzubauen; Eisen findet sich sogar viel, vielleicht können wir auch auf Kupfer hoffen. Ob jedoch das schon vor Jahrhunderten ersehnte Gold, das hellgelbe afrikanische Gold, sich finden dürfte, glauben wir nicht.

Unter den gesuchtesten Tauschartikeln steht der Baumwollst off an erster Stelle. Nicht aber auf die Buntfarbigkeit, wie uns immer fälschlich gelehrt wurde, legt der Neger Wert, sondern er mustert mit Kennerblicken seine Haltbarkeit und sein Gewebe.

Mit Perlen als Wertmesser lassen sich die besten Geschäfte abwickeln. Die verschiedenen Perlen richten sich nach Gegenden, in der einen werden rote in der andern blaue bevorzugt. Am gangbarsten sind allgemein die weißen und die dunkelroten Perlen. Viele, wohl die meisten, stammen aus Gablonz und anderen böhmischen Orten. Aber auch blaue eckige Perlen an 
Schnüren bilden Handelseinheiten, dabei läßt sich der Neger nicht verdrießen, sie einzeln nachzuzählen. Wenn man auch noch so treibt, er wird sich zum Abzählen unbedingt Ruhe gönnen, da er der Meinung ist, daB man ihn tibervorteilen will.

Gern wird sodann Messingdraht genommen, der zu Arm- und Beinspangen, aber auch als Hals- und Ohrschmuck Verwendung findet. Messingstäbe, jeder wiegt durchschnittlich $200 \mathrm{~g}$, gelten teilweise auch als Geld; der einzelne Stab wertet 50-80 Pfennig. Kupfer, ebenfalls ein Wertmesser, kommt herauf aus dem Süden, meist in den eigenartig kreuzartig geschmolzenen Formen zum bequemeren Transport.

Einen begehrenswerten Handelsartikel bildet auch das Salz, doch übertrifft es nicht das Pulver, das überall gern an Geldesstatt genommen wird, ebenso wie Schießwaffen, seien es auch noch so veraltete Modelle.

Bezahlung in Gin, einem ebenso gemeinen wie gemeingefährlichen Rum, ist jedoch des Glückes Höchstes. Stark begehrt ist dieser Branntwein; er kommt gröBtenteils aus Hamburg und ist in entsprechenden Gefäßen so verpackt, daß immer zwei Flaschen eine Trägerlast ausmachen. Der Gin geht jedem Neger über alles, darum kann man sich auch den Ruin des Schwarzen mit dem Fusel ausmalen. Der Branntwein muß stets mit Wasser vermischt verabreicht werden, meist sorgt schon ein Zwischenhandel für gehörige Verdünnung. Wie leicht ist es gesagt: Gebt keinen Rum (und welche Sorte erst!). Der Neger liefert aber einfach auch dem Reisenden keine Lebensmittel. Was aber soll dann dieser machen? $\mathrm{Zu}$ den notwendigsten Tauschmitteln zählt der Gin nun einmal und - steht besonders hoch im Preise.

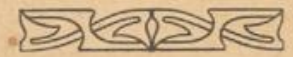




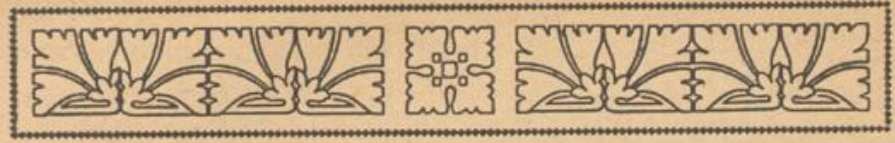

\section{V.}

00000000 ie Erwerbsmöglichkeiten sind im neuen Kolonialgebiet nun sehr verschieden. Wir wollen einmal eigene Erfahrung sprechen lassen, sodann aber alles für die Praxis des 00000000
was uns solonisten und Reisenden zusammentragen, Gelehrten und Praktikern mit großem Lehrgeld gesammelt wurde.

In vielen Gegenden gibt es nur wenig Lebensmittel, überall sind sie schwer aufzutreiben. Nicht selten mangelt es sogar an Brennholz, obwohl der Wald unmittelbar dabei liegt. Für den Europäer stellen sich auch sonst noch die vielerlei Kleinigkeiten ein, auf die er nicht gern verzichtet.

Auf gute Waffen haben Ansiedler zu sehen; Jagdartikel werden je nach dem Lande am geeignetsten von Spezialfabriken zusammengestellt, wir könnten dazu Albrecht Kindt, Hunstig 59 bei Dieringhausen empfehlen.

Der Einwanderer nach Deutschkongo wird sich, wenn er über wenige Geldmittel verfügt, sehr mit den Behörden, den Kolonisten, den Missionaren und den Eingeborenen auf guten $\mathrm{FuB}$ zu stellen versuchen, denn in dem neuen Gebiete treffen bis zur völligen Ordnung Faktoren zusammen, die ihm jedes ersprießliche Arbeiten werden verleiten können.

Das Klima setzt dem Kolonisten auch zu; viele 
laufen skelettartig abgemagert herum und ihre Gesichtsfarbe ist wachsgelb durch Anämie oder Ikterus. Darum soll man vorsichtig im Genießen sein. Fette Speisen muß man meiden, auch den Alkoholgenuß einschränken. Hellfarbige Bekleidung, luftige Flanellanzüge sind ratsam.

Am gefährlichsten, hinsichtlich der Malariainfektion, sind die Abend- und die Nachtstunden, daraus ergibt sich auch, daß die auf Schiffen, Hulks wohnenden, dort auch schlafenden, aber mit Landdienst beschäftigten Personen weniger unter dem Fieber zu leiden haben als Landbewohner.

Faßt man alles zusammen, so kommt eine ganze Epistel voll „Das darfst Du nicht“ zusammen. Martin sagt dem Händler und Kolonisten in „Influences of tropical Climates", wie er sich zu verhalten hat und zwar:

Sorgfalt in Bezug auf Nahrung, Kleidung und Leibesbewegung sind für die Erhaltung der Gesundheit viel wesentlicher als ärztliche Behandlung.

Der richtige Weg, um Krankheiten zu entgehen, ist die Beobachtung strengster Mäßigkeit und die Verringerung der Hitze auf jede mögliche Weise.

Nachdem die Hitze den Körper für die Krankheit vorbereitet, hat plötzlicher Eintritt von Kälte den allerschlimmsten Einfluß auf die menschliche Konstitution.

Die große physiologische Regel, um die Gesundheit in heißen Klimaten zu bewahren, ist, den Körper kühl zu halten, erhitzende Getränke zu vermeiden.

Ein kaltes Bad ist nach jeder großen Ermüdung des Körpers und des Geistes Tod.

Ausschweifungen sind von viel gefährlicherer und zerstörenderer Wirkung als in Europa.

Eine große Menge tierischer Nahrung erhitzt das Blut, anstatt Kräfte zu geben, schwächt den ganzen Körper.

Brot ist eins der besten Nahrungsmittel; Reis und 
getrocknete Erbsen sind gesund und nahrhaft; Gemüse, wie Möhren, Rüben, Zwiebeln, Kohl usw. sind für gute Gesundheit von Wichtigkeit.

Früchte sind nur in reifem und gesundem $\mathrm{Zu}$ stande nützlich. Spirituosen sind zu verdünnen.

Europäer können bei genauer Vorsicht und hygienischer Beobachtung auch in den Tropen lange leben.

Nun, wir sind keineswegs mit seinen Ausführungen zufrieden, die Ansichten gehen eben vielzusehr auseinander. Jedenfalls aber ist MäBigkeit und MäBigung in allen Dingen eine große Notwendigkeit, um in den Tropen gesundheitlich $\mathrm{zu}$ bestehen.

Die leichteste Weise im zentralen Afrika Geld zu machen, ist das Tauschgeschäft. Durch Tauschhandel kamen noch alle Händler zu Reichtum, obwohl sie manches Mal oder ziemlich "immer" natürlich ihren Vorteil besonders groß schrieben, denn wo der Tauschhandel aufhörte, hörte auch das schnelle Reichwerden auf. Dazu freilich tritt oft noch eine hohe Besteuerung, hohe Zölle. Eine Besteuerung darf nicht so hoch sein, ehe sich eine Kolonie nicht wirklich rentiert.

Jedoch verwerfen wir, aufs Ungewisse Handel treiben zu wollen. Es würde sich sonst bitter rächen. Nicht weniger kommt es auch auf den Charakter des Reisenden an. Geduld und Kaltblütigkeit sind zwei Kardinaltugenden, die man von jedem Ansiedler und Händler unbedingt verlangt.

Jeder Afrikareisende wie Kolonist muß sein Unternehmen auf friedliche Weise zu fördern trachten, hingegen müssen gegebenenfalls Hartherzigkeit und strenge Zucht angewendet werden können.

Anfänglich wird durch Wareneintausch das Geschäft noch ziemlich leicht gemacht, während später der Austausch sich wohl nur gegen Bargeld bewerkstelligen lassen wird, denn nur wenig lernt der Neger 
so schnell bemeistern als den Gebrauch, den Wert des Geldes.

Das neue Gebiet ist zwar nur etwas für unternehmungslustige Leute, denen irgendwie Kapital zur Verfügung steht oder die doch durch Verbindungen solches unter Umständen flüssig machen können. Ohne Verbindungen wird das schwer halten, da das deutsche Kapital praktischer geworden ist und sich nicht mehr durch Kolonialoptimismus blenden läßt. Wo aber positive Arbeit geleistet wird, muß das Kapital gut arbeiten, nicht zuletzt aber wird das im neuen Deutschkongolande möglich sein.

Für das neue Kongogebiet aber möchten wir in erster Linie erwähnen, daB es auf charakterfeste Ansiedler ankäme, die den Handel erst in zweite Linie stellten und in erste die Kolonisation, den Landanbau. Sich selbst ernähren würde dem nicht schwer fallen, der sich dem Klima anpassen könnte. Einer kann sich dort leichter durchbringen, wenn er sich anzupassen versteht, als große Unternehmungen, die rentieren sich leider nicht recht.

Auch französische Aktien stehen an der Börse augenblicklich kläglich, auch dort zeigt sich eine auffällige Müdigkeit im Handel mit Kolonialwerten. Es wurde freilich viel versprochen und wenig gehalten, manches investierte Kapital wird vielleicht auch nie das Kongogebiet gesehen haben, sondern in Paris geblieben sein, ganz abgesehen, daß einige Unternehmen in großer Unerfahrenheit Ansiedlungen bauten, die sich niemals rentieren können. Der Kolonialrummel hat auch die Ignorantesten zu Kolonialverwaltern gemacht, wenn wir auch zugestehen wollen, daß die äußerst geschickte Hand eines Stanley, Brazza, Peters zu solchen Gründungen notwendig werden muß, die Ansiedlungen lebens- und einbringfähig zu halten.

Mit einen weitern Einfluß hat man in den Farbigen 56 
zu kämpfen, die nicht Bantu sind und die aus früheren Expeditionen in den Gebieten zurückblieben, meist Mohammedaner. Der Islam mit seinen aufreizenden Forderungen nach dem Dschihad, dem hl. Krieg fördert keine Kolonie. Wir finden hier wenige Sudanesen, Hausasoldaten und Zansibarleute, die dem Reisenden und Kaufmann das Leben schwer zu machen wissen.

Für den Ansiedler allein ist es sehr schwer, Verbindungswege zu schaffen, obwohl schon Brazza das als Hauptnotwendigkeit hinstellte. Darum versuchten schon die Franzosen seit dem Anfang der neunziger Jahre vom Stanley-Pool und mittlern Ubangi nach ihrem Kongohinterland, nach dem Schari und Nil vorzudringen.

Als erstes wird man dann den Wald niederhauen müssen, den Erdboden aufgraben, das Sumpfland entwässern, fürwahr ein schwieriges Mühen. Technische Schwierigkeiten zum Wegebau sind hier natürlich leicht zu überwinden (sagt Lenz), aber viel Ausdauer und Geduld gehört doch dazu. In der Trockenzeit pflegt man auch das Gras abzubrennen, um so leicht den Boden zu düngen.

Auf vorbereitetem Boden wachsen die meisten unserer Kulturpflanzen gut, es gedeihen Zwiebeln, Möhren, Kohl, Rüben, Bohnen, Erbsen, Tomaten; ferner werden Orangen- und Zitronenbäume (Aurantiaceen) wohl auch erfolgreich angebaut werden können, doch wird sich der Transport nach Europa selbst nach Inbetriebsetzung guter Frachtverbindungen kaum lohnen.

Die Ananas wächst hier wild und ihre Früchte sind ungenieBbar, doch kommen auch die veredelten Ananaspflanzen fort. Aber Ananas anzupflanzen hat keinen großen Wert, da die Aussichten zum Absatz heute noch schlecht liegen und erst die Zukunft ergeben muß, ob man diese Frucht mit größerm Nutzen kultivieren kann. Prächtig gedeihen Kürbisse in verschiedenen Arten und Melonen. 
Zuckerrohr trifft man seltener; wo Zuckerrohr sich findet, sind meist auch Nilpferde, sagt Stanley und das trifft in einzelnen Kongogegenden auch zu. Die Kokuspalme muß angebaut werden.

Tabak gibt es auch, er ist freilich meist zu scharf, wird daher wenig gebaut, doch käme es auf Kulturversuche an. Hingegen wird sich der Anbau von Vanille lohnen. Wo die Franzosen sich seßhaft gemacht haben, beginnen sie bekanntlich bald auch mit Kulturversuchen der Vanille. Sie wirkt giftig und zwar erkennbar durch Entzündung, Ausschlag, Schwindel. Zur Haltbarkeit überstreicht man die Vanilleschoten vielfach mit dem Oele von Anacardium accidentale (Akajubaum), dem Cardol.

Spanischer Pfeffer und andere Gewürzpflanzen erweisen sich lohnend zum Anbau. Der spanische Pfeffer, Capsicum annuum findet in den Tropen allgemeine Verwendung. $\mathrm{Zu}$ viel genossen ruft er durch seine Flüssigkeit, das Capsicol Vergiftungserscheinungen hervor. Man trifft auch Baumwollekulturen, wenn diese auch meist ausgehen von Gosypium arboreum, der dort zwar wild wächst, aber auch nur minderwertiges und geringes Rohmaterial liefert, das wenig im Tauschverkehr verlangt wird.

Kaffee und zwar Coffea liberica dürfte gut gedeihen und in mindestens gleichwertiger Qualität wie etwa der der portugiesischen Insel Sao Thomé. Apothekerpflanzen sind anbaufähig und mit Erfolg zu kultivieren, nicht zuletzt möchte ich auf Datura stramonium, den Stechapfel hinweisen.

Mit der Viehzucht ist es schlechter bestellt. Rinder, wie sie weiter südlich vorkommen und als Reittier benutzt werden, ließen sich vielleicht auch hier züchten, sofern die Tsetsefliege ausgerottet oder doch erfolgreich bekämpft werden kann.

Ziegen und Schafe sind in diesem Gebiete wohl 
die einzigen dankbaren Nutztiere, daneben noch das graue afrikanische Schwein.

Versuche mit Zebrazüchtung und Zugtierverwendung kämen vielleicht in Frage. Im Allgemeinen liegen hierfür überhaupt noch zu wenige praktische Erfahrungen vor, Strauße und Kamele kommen für diese Gebiete wohl fast garnicht in Betracht.

Wir haben in dem erworbenen Kongogebiet mit einer erheblichen Anzahl größerer französischer Gesellschaften zu rechnen. Insgesamt kämen ohne Privatniederlassungen etwa 33 in Betracht. Von diesen scheiden hingegen etwa 20 insofern aus, als sie im deutschen Kongolande nur Zweigniederlassungen, Einkaufsstellen u. ä. haben, 13 hingegen sind ganz in unserm neuen Gebiet interessiert. Als besonders große Unternehmungen kommen jedoch nur wenige in Betracht. Die französischen Gesellschaften haben auf irgendwelche Entschädigungen von Seiten der französischen Staatsregierung natürlich keinen Anspruch, da nach dem staatlichen Uebereinkommen, das die Gesellschaften einfach werden anerkennen müssen, die Oberhoheit von Frankreich auf Deutschland übergeht.

Die wirtschaftliche Entwicklung durch Konzessionsgesellschaften war unter der französischen Regierung eigentlich undenkbar oder die schweren Verpflichtungen mußten irgendwie anders beigetrieben werden. Frankreich verlangte bei Konzessionserteilung $u$. a. französisches Kapital, Vorherrschaft der Franzosen in Aufsichtsrat und Verwaltung, Gewinnbesteuerung bis $15 \%$ für den Staat, Wegebau, Schiffahrtsunterhaltung, Aufforsten von Kautschukpflanzen. Die portugiesischen Handelsgesellschaften und Agenten arbeiten mit hohen Prozenten, sodaß sie sich in kurzer Zeit ein bedeutendes Vermögen erwerben.

Die französischen Gesellschaften mit ihren Kon- 
zessionen hat freilich die deutsche Regierung mit übernommen. Das Kolonialamt schreibt zwar darüber: Für uns kommen in der Hauptsache nur zwei Konzessionsgesellschaften in Frage.

a) In dem an Südkamerun angrenzenden Dreieck die Gesellschaft Ngoko-Sanga. Diese Gesellschaft ist insofern in einer mißlichen Lage, als sie den größten Teil ihres Konzessionsgebietes auf dem Wege über den Sanga wegen der großen örtlichen Entfernung nicht gewinnbringend ausbeuten kann. Der natürliche Abfuhrweg für die größere westliche Hälfte ihres $\mathrm{Ge}$ bietes führt nach den örtlichen geographischen Verhältnissen an die jetzige Kameruner Südküste. In der Tat ist das Konzessionsgebiet, ehe die Kameruner Südgrenze in natura festgelegt war, von Norden her durch die Kameruner Südfirmen mit ausgebeutet worden. Die Gesellschaft sah sich im vorigen Jahre veranla $\beta$ t, eine Interessengemeinschaft mit den deutschen Firmen jenseits der Kamerungrenze anzubahnen. Die damaligen Verhandlungen, die von deutscher beteiligter Seite mit Interesse aufgenommen wurden, zerschlugen sich alsdann aus Gründen, die von den Willen der beiden Parteien unabhängig waren. Es ist anzunehmen, da $B$ die Verhandlungen, sobald das Konzessionsgebiet der Gesellschaft Ngoko-Sanga deutsch wird, wieder aufgenommen und zu einem Zusammenarbeiten führen werden.

b) Das Gebiet zwischen Sanga und Ubangi ist das Konzessionsgebiet der Compagnie Forestière SangaOubangi. Sie ist im Jahre 1911 aus elf Gesellschaften entstanden, die sämtlich durch Vertrag mit dem französischen Staate in eine Abänderung ihrer Konzessionsbedingungen eingewilligt hatten. Durch diese Abänderung ist das Konzessionssystem erheblich gemildert worden. Das allgemeine Kautschukmonopol erlischt 1919 anstatt 1929. Die Grundrechte der Eingeborenen 
und ihre gute Behandlung durch die Gesellschaften sind in erhöhtem Maße gesichert worden. Am Ende der Konzessionsdauer (1929) werden die Konzessionsgesellschaften Eigentümer lediglich desjenigen Teiles ihres Gebietes, das sie tatsächlich in Kultur genommen haben, und das wird voraussichtlich nur ein kleiner Prozentsatz ihres ursprünglichen Konzessionsgebietes sein. Die Gesellschaft selbst schätzt diesen Teil auf höchstens 5000 Quadratkilometer bei einer Größe des gesamten Konzessionsgebietes von rund 170000 Quadratkilometer. Alles übrige Land fällt dann in das Eigentum des Fiskus zurück. Wenn die jetzigen Konzessionsbestimmungen so gehandhabt werden, wie sie lauten und gemeint sind - und daß dies geschieht, wird die deutsche Regierung durchsetzen - , dürfte die Sicherheit geboten sein, daß dem Lande die reichen wirtschaftlichen Werte, die die Natur ihm gegeben hat, trotz des oben bereits bedauerten Konzessionssystems erhalten bleiben.

Die Schwierigkeiten, die daraus sich ergeben, daB die neue Grenze das Konzessionsgebiet durchschneidet, werden durch eine besondere Vereinbarung mit der franzősischen Regierung aus dem Wege geräumt werden.

Es soll hier noch einmal betont werden, daß die Konzessionen die Bodenschätze nicht mit umfassen. Die Gesellschaft Sanga Equatoriale am rechten Unterlauf des Sanga scheint nur noch zu vegetieren, Kurs in den "Annales Coloniales" vom 17. Oktober 1911 gegenüber den Kursen der übrigen Gesellschaften außerordentlich niedrig (15). Die Compagnie Commerciale de Colonisation du Congo Français und manche andere ist in diesem Kurszettel überhaupt nicht mehr enthalten.

Viele Aktien von Kongogründungen sind nie ausgegeben und gehandelt, sondern ihr Unternehmen hat erst gar nicht begonnen, im andern Falle wäre die 
Entrüstung über das neue deutsche Kongogebiet in Frankreich noch wesentlich geringer.

Im "Temps" veröffentlicht der Aufsichtsrat der Sanga-Ubangi-Gesellschaft einen offiziellen und feierlichen Protest gegen die mögliche "Sezierung“ des von der Gesellschaft kolonisierten Gebietes. Es wird darin lebhaft gegen die Unterschätzung der kolonialen Landschaften protestiert, wie sie jetzt in der gouvermentalen französischen Presse und der deutschen Presse beliebt sei. Es heißt in der Erklärung: Die Summe der Kapitalien, die im Kongo engagiert sind, erreicht 55 Millionen Francs, von denen 38 Millionen durch Frankreich aufgebracht sind und in Frankreich gearbeitet haben. Der Handel der Kolonie, der vor Gründung der Gesellschaft stagnierte und 11 Millionen Francs nicht überschritt, hat sich im Laufe von zehn Jahren verdreifacht, und der Anteil der Kongogesellschaften übersteigt 55 Prozent. Dank der Arbeit der verschiedenen Kolonialgesellschaften, die im Jahre 1899 das Land auf dreißig Jahre pachteten, ist der Kongokautschuk auf den europäischen Plätzen eingebürgert. Mehr als 9000 Tonnen sind seit Beginn der Kolonisation ausgeführt worden, und die Stadt Havre ist für diese Handelsbeziehungen ein außerordentlich wichtiger Markt geworden. Auch hat die Gesellschaft das Budget der französischen Kongokolonie durch eine große Reihe von Einnahmen, die sie ihm sicherten, belebt, durch die Ein- und Ausfuhrzölle, durch Benefizien und Stempel. Gerade jetzt ist der Augenblick gekommen, in dem die Interessen der verschiedenen Gesellschaften sich vereinten und die Früchte einer langjährigen $\mathrm{Ar}$ beit gepflückt werden könnten.

Die Deutsche Kongo-Liga gegen das französische Kolonisations-System am Kongo hat zu dem KongoAbkommen folgende Resolution gefaßt: Durch das Marokkoabkommen vom 4. November ist Deutschland

62 
in den Besitz mehrerer hunderttausend Geviertmeter Landes gelangt, in denen auf Grund der Berliner Kongoakte von 1885 völlige Handelsfreiheit herrschen soll. Der größte und wertvollste Teil dieser Gebiete liegt aber heute in den Händen französischer Konzessionsgesellschaften, deren monopolistische Rechte jeden Freihandel und eine gesunde Eingeborenenpolitik ausschliessen. Fürst Bismarck, der Einberufer der Kongokonferenz, sah die beiden Grundprinzipien der Kongoakte in einer völlig freien Handelsbetätigung und der Pflege der Eingeborenenbevölkerung. Die koloniale Entwicklung der letzten 35 Jahre hat seinen Forderungen recht gegeben: Die dem Freihandel geöffneten afrikanischen Kolonien Deutschlands, Englands und Frankreichs, in denen die Eingeborenen ihr natürliches und in der Kongoakte als selbstverständlich vorausgesetztes Recht auf freie Verfügung über die Bodenprodukte erhalten geblieben ist, haben sich großartig entwickelt, während die Gebiete mit privilegiertem Handel in ihrer Entwicklung zurückgeblieben sind. In den Freihandelgebieten geht die materielle und sittliche Hebung der Eingeborenen mit dem Blühen des Handels Hand in Hand, dagegen hat das System im französischen Kongo zu einer skrupellosen Aussaugung des Landes und Ausbeutung der Eingeborenen geführt und dadurch auch die Ausbreitung der Schlafkrankheit begünstigt.

Die Deutsche Kongo-Liga, deren Aufgabe in der Förderung des Wohles der Eingeborenen und in der Verteidigung deutscher Vertragsrechte in Zentralafrika besteht, protestiert gegen das Fortbestehen dieser $\mathrm{Zu}$ stände, die in striktem Gegensatz zu den Festsetzungen der Kongoakte stehen. Sie bittet den Reichskanzler, in dem deutsch gewordenen Teil des bisherigen Französisch-Aequatorialafrika die bestehenden Privilegien zu beseitigen, die Eingeborenen von der Verpflichtung, für die Gesellschaften zu arbeiten, zu befreien, ihnen 
die freie Verfügung über die Produkte des Bodens zurückzugeben und durch hygienische und soziale Maßregeln für die Besserung des Loses der Eingeborenen zu sorgen, da ohne eine kräftige und zahlreiche Bevölkerung eine Entwicklung des Landes nicht möglich ist.

Diese Ausführungen sind von großem Werte und der Liga müssen wir dafür Dank wissen, denn sie packt von vornherein das Uebel an der Wurzel. Wir müssen ihr daher Erfolg wünschen.

Doch nun kommen wir allmählich zum SchluB. Nehmen wir alles mit objektivem Betrachten, so haben wir viel Gutes, leider aber auch manches Böse zu erwarten. Lenz, der Leiter der österr. Kongoexpedition sagt treffend: Das ganze Kongobecken, wie überhaupt das ganze tropische Afrika, ist und bleibt einmal ein für Europäer ungesundes und gefährliches Land, man mag das zu beschönigen suchen wie man will, es nützt alles nichts. Ich halte jeden Versuch, auch nur ein Wort zu Gunsten des Klimas zu sagen, für gewissenlos und verbrecherisch, nur geeignet, unerfahrene Leute hierher zu locken, wo sie neben Enttäuschungen aller Art auch noch Leben und Gesundheit aufs Spiel setzen; es ist ganz gleichgiltig, ob das Land am Meere liegt oder im Innern, ob der Platz hoch oder tief gelegen ist, es ist und bleibt ein ungesundes Klima und jeder, der mit heiler Haut diese Länder verläßt, kann von Glüick sagen.

Ein Träger- und Arbeitermangel wird dem neuen Ansiedler auch fühlbar werden, dazu kommt für ihn selbst noch das Heimweh hinzu, das zu einem nervősen Leiden werden kann, wenn - alles nicht klappt. Mutlosigkeit aber ist die erste Schaufel zum Grabe einer Ansiedlung.

Da Deutschland von vornherein nicht große Mittel für das koloniale Neuland ausgeben dürfte, ist die Ansiedlung keinesfalls ratsam. Man wird zunächt erst 
abwarten müssen, was kommt, ehe man sich bindet. Nicht zuletzt dürften Reisen in das Gebiet, in dem man sich anzusiedeln beabsichtigt, notwendig sein, dabei lernt man die Umgebung am besten kennen. Es geht hier nicht, daß man Ort und Stelle in Deutschland ausknobelt; hier heißt es, zunächst von BelgischKongo rekognoszierend vorzudringen. $\mathrm{Ob}$ aber die Arbeit und Mühe den Verdienstpreis erringen, das ist eine Frage der Zeit - ein Wechsel auf die Zukunft.

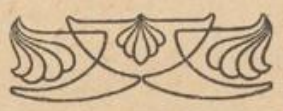




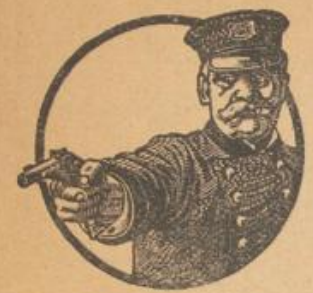

\section{Munition-, Jagdantikel} sowie die bekannten automatischen

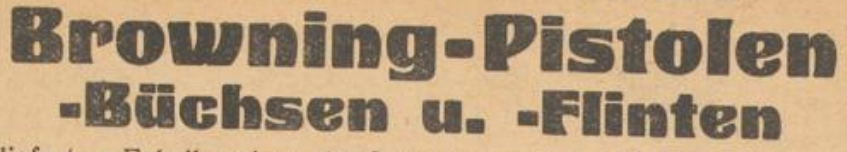

liefert zu Fabrikpreisen die Generalvertretung für Deutschland: Albrecht Kind, Hunstig 59 b. Dieringhausen

Fabrik u. Lager von Jagdgeräten u. Waffen.

Export. $\circlearrowright 0$ Lieferung nur an Wiederverkäufer. $\infty 0$ Export. Preisliste gegen Einsendung von $50 \mathrm{Pfg}$ für Porto zu Diensten.

Jedem nützlich!

Jedem nützlich!

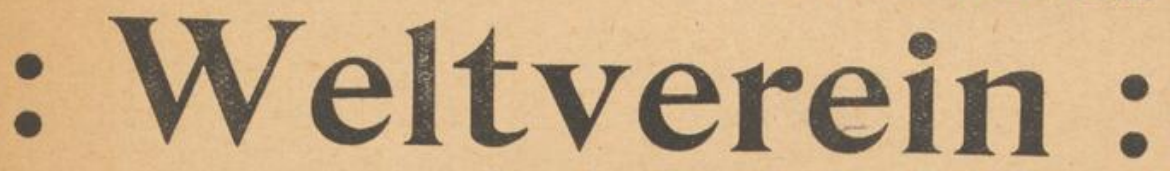

Keine Aufnahmegebühr. $\circ \infty$ Prospekt und Zeitung gegen

Einsendung einer 20 Pfennig-Marke franko von der

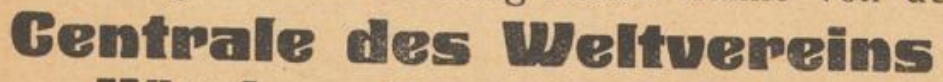
Münehen, Auenstralie 64, l.

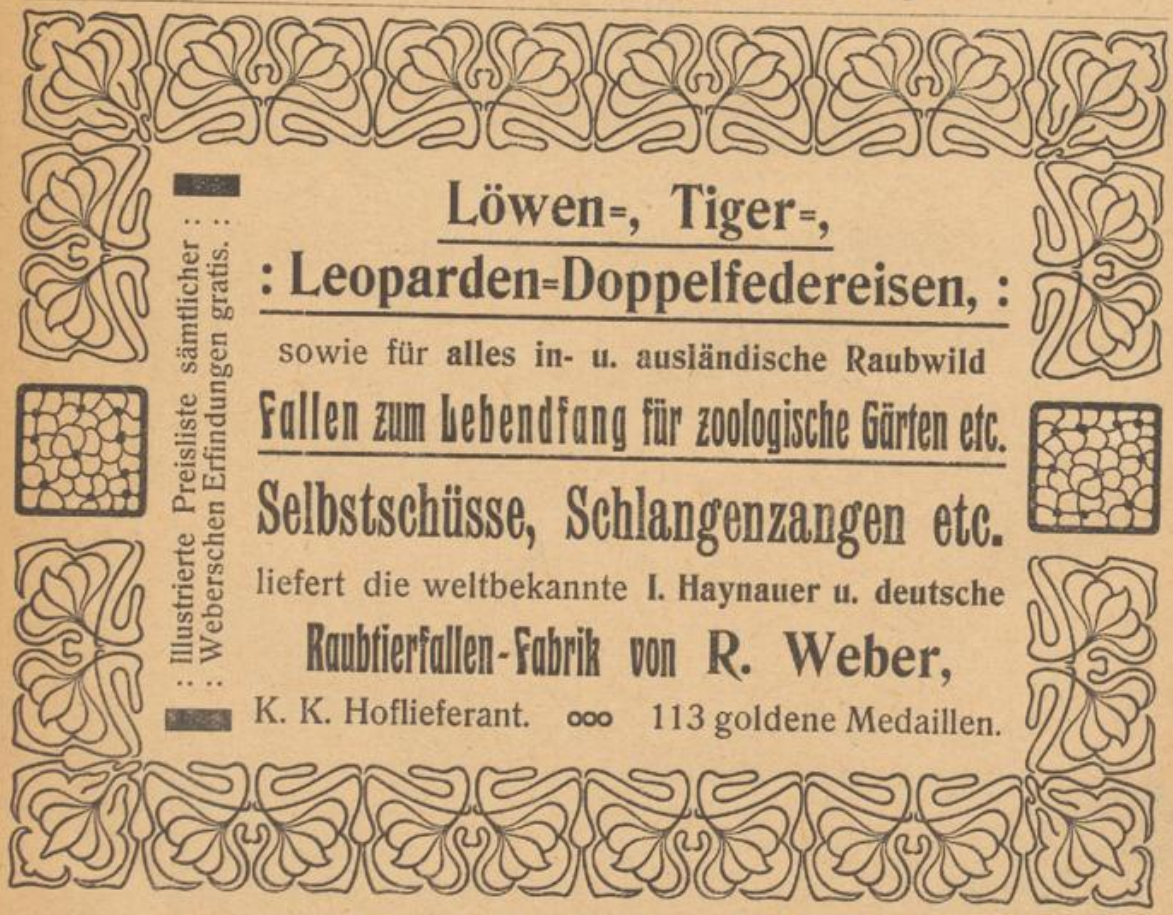


UB Frankfurt

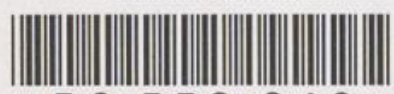

56553240 


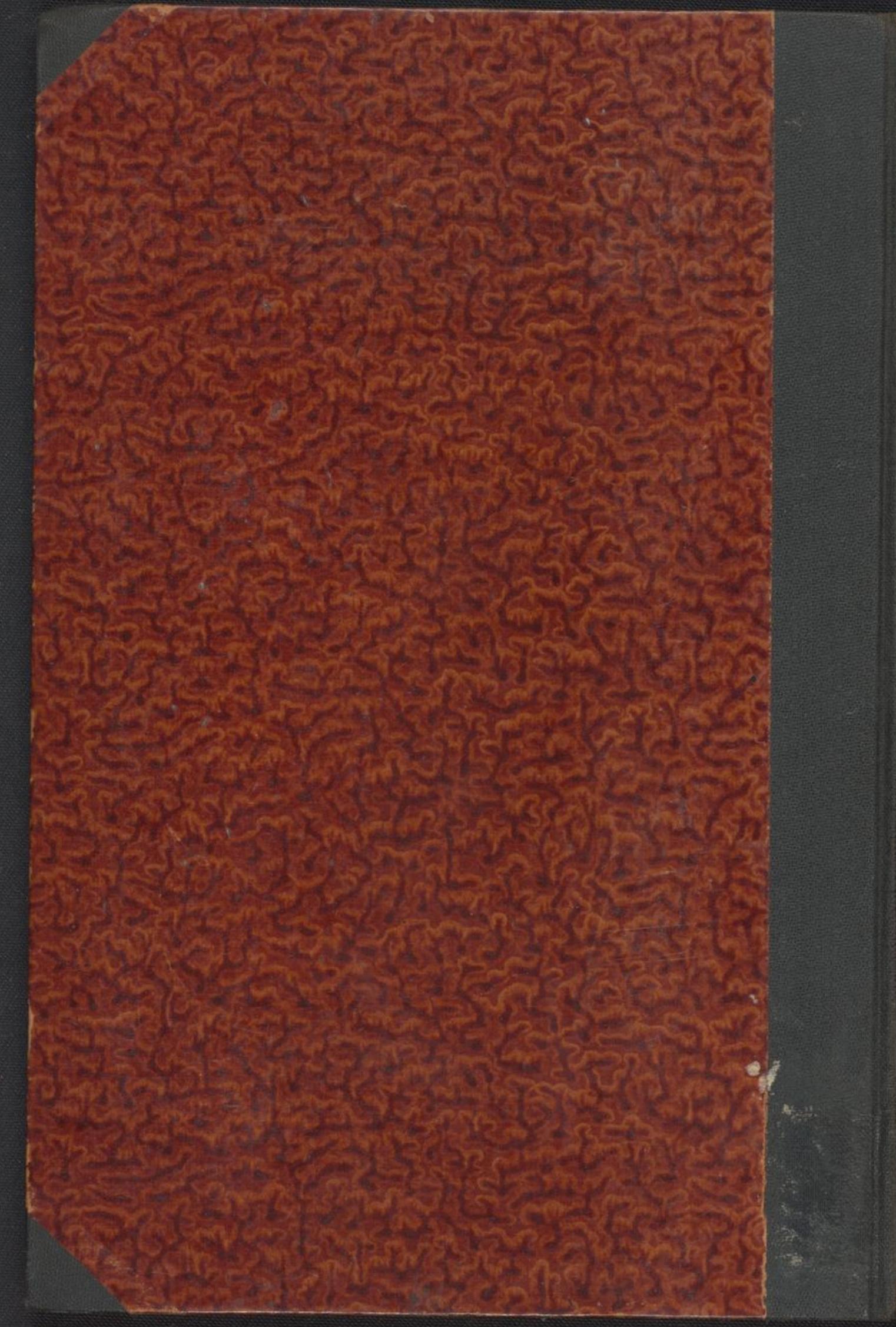

